\title{
Martin Zimmermann
}

\section{Die Repräsentation des kaiserlichen Ranges}

Die Repräsentation des kaiserlichen Ranges kann vor dem Hintergrund der antiken Zeugnisse aus verschiedenen Blickwinkeln beschrieben werden. Die antike Literatur etwa bietet eine Vielzahl von Hinweisen darauf, wie die soziale und politische Stellung der einzelnen Kaiser in der Öffentlichkeit inszeniert wurde. Ferner gewährt die reiche Erhaltung von Kaiserstatuen oder Staatsreliefs die Möglichkeit, die Indizien für die Darstellung des Ranges im Bildnis zusammen zu tragen. Gleiches gilt für die Inschriften, in denen nicht nur eine umfangreiche Titulatur, sondern weitere Formen der Selbstdarstellung in ihrer historischen Entwicklung greifbar sind. In einer systematischen Zusammenstellung solcher Quellen und Monumente ließe sich zeigen, wie die äußere Erscheinung der Herrscher in Auftreten, Kleidung, Insignien oder Titulatur gestaltet wurde und sich im Laufe der Zeiten wandelte.

Andreas Alföldi hat hierzu seit Mitte des 20. Jahrhunderts bereits grundlegende Studien vorgelegt $^{1}$. Seitdem ist freilich nicht nur das Material, das sich für die Aktualisierung seiner Arbeiten heranziehen ließe, immens gewachsen, sondern anhand einzelner Monumentgattungen oder Bildelemente konnte zudem gezeigt werden, welche differenzierten Ergebnisse zu erzielen sind, wenn einzelne Bildformeln, Insignien oder Wertvorstellungen im Detail erforscht werden².

\footnotetext{
${ }^{1}$ Einige Studien sind zusammengefasst in dem Band Andreas Alföldi, Die monarchische Repräsentation im römischen Kaiserreich (Darmstadt 1970). Vgl. ferner Frank Kolb, Zur Statussymbolik im antiken Rom, in: Chiron 7 (1977) 239-259 und zur Unterscheidung zwischen formellen und informellen Symbolen für die vorchristliche Zeit und den Osten Hartmut Blum, Purpur als Statussymbol in der griechischen Welt (Bonn 1998) bes. 1-19. Siehe ferner den Überblick bei Peter Scholz, Zur öffentlichen Repräsentation römischer Senatoren und Magistrate, in: Tobias L. Kienlin (Hg.), Die Dinge als Zeichen. Kulturelles Wissen und materielle Kultur (Bonn 2005) 409-431.

${ }^{2}$ Siehe nur Hans R. Goette, Mulleus-Embas-Calceus, in: JdI 103 (1988) 401-464; Thomas Schaefer, Imperii Insignia. Sella curulis und Fasces (Tübingen 1989); Jutta Rumscheid, Kranz und Krone. Zu Insignien, Siegespreisen und Ehrenzeichen der römischen Kaiserzeit (Tübingen 2000); Marianne Bergmann, Die Strahlen der Herrscher. Theomorphes Herrscherbild und politische Symbolik im Hellenismus und in der römischen Kaiserzeit (Mainz 1998); siehe auch den Überblick bei dies., Repräsentation, in: Adolf H. Borbein u.a. (Hg.), Klassische Archäologie. Eine Einführung (Darmstadt 2000) 166-188. Siehe jetzt auch die methodisch wegweisende Studie von Birgit Bergmann, Der Kranz des Kaisers. Genese und Bedeutung einer römischen Insignie (New York u.a. 2010) 209-212 (zu „Chancen und Grenzen der ,Realienforschung““). Ein gutes Beispiel für die Schwierigkeiten, am konkreten Objekt einer Statue die Standeszeichen eines römischen Bürgers unzweifelhaft festzustellen, siehe z.B. Klaus Fittschen, Der, Arringatore', ein römischer Bürger?, in: RM 77 (1970) 177-184. Es gilt weiterhin die Feststellung Kolbs, Statussymbolik (wie Anm. 1) 244: „Es fehlt nicht nur eine zusammenfassende Darstellung mit einer klaren Klassifizierung der Symbole, sondern überhaupt eine eingehende Behandlung der Geschichte der einzelnen Statusabzeichen einschließlich ihrer gesellschaftlichen und politischen Funktion.“
} 
Da eine Gesamtschau nach wie vor ein Desiderat der Forschung darstellt ${ }^{3}$ und an dieser Stelle selbstverständlich nicht nachgeholt werden kann, soll hier das Augenmerk auf einen Aspekt der Repräsentation gerichtet werden, der in den einleitenden Sätzen bereits angeklungen ist und auch in den Überlegungen Alföldis einen zentralen Platz einnimmt. Die Repräsentation des kaiserlichen Ranges soll als komplexer Kommunikationsprozess verstanden werden, in den neben den einzelnen Kaisern die römische Gesellschaft in ihrer Gesamtheit und zu einem Teil auch die Provinzialen eingebunden waren ${ }^{4}$. Repräsentation soll ungeachtet der alles beherrschenden Stellung des Kaisers demnach als Vorgang einer permanenten Verständigung darüber verstanden werden, wie der Rang adäquat in unterschiedlichen Situationen und Kontexten wiedergegeben werden sollte. Diese Situationen und Kontexte selbst, von denen in jüngster Zeit etwa die salutationes und convivia Aufmerksamkeit gefunden haben ${ }^{5}$, bieten in der Interaktion zwischen Kaiser und Anwesenden die Möglichkeit, das eigene Verständnis der Herrschaft zu dokumentieren, die eigene Machtstellung zu sichern, zumindest jedoch die Bereitschaft zum Dialog zu signalisieren und somit das objektiv bestehende Machtgefälle zu entschärfen.

\section{Die pompa funebris für Pertinax als Inszenierung des kaiserlichen Ranges}

Öffentlichkeit spielte bei einer auf Anwesenheit der unterschiedlichen Adressaten orientierten Herrschaftsrepräsentation naturgemäß eine zentrale Rolle. Daher kann man sich der komplexen Fragestellung gut nähern, wenn man als Ausgangspunkt Festlichkeiten wählt, an denen viele Gruppen der Gesellschaft teilnahmen, in denen aber der Kaiser im Mittelpunkt stand. Besonders ausführliche Berichte besitzen wir von den Feierlichkeiten, die mit der Bestattung des Herrschers verbunden waren. Nicht allein die Amtseinführung, nicht die adventus- sowie profectus-Zeremo-

\footnotetext{
${ }^{3}$ Siehe zum Kontext der Rangrepräsentation die von Pierre Bourdieus soziologischer Kategorie des Prestiges bzw. des symbolischen Kapitals inspirierte Studie von John E. Lendon, Perceptions of Prestige and the Working of Roman Government (Diss. Oxford 1991), die begrifflich neutraler als John E. Lendon, Empire of Honour. The Art of Government in the Roman World (Oxford 1997) publiziert wurde.

4 Siehe hierzu ausführlich Gregor Weber, Martin Zimmermann, Propaganda, Selbstdarstellung und Repräsentation. Die Leitbegriffe des Kolloquiums in der Forschung zur frühen Kaiserzeit, in: Dies. (Hg.), Propaganda - Selbstdarstellung - Repräsentation im römischen Kaiserreich des 1.Jahrhunderts n. Chr. (Stuttgart 2003) 11-40; Gunnar Seelentag, Taten und Tugenden Traians. Herrschaftsdarstellung im Principat (Stuttgart 2004) 35 u.ö. Die Überlegungen von Karl A.E. Enenkel, Ilja L. Pfeijffer, Introduction, in: Dies. (Hg.), The Manipulative Mode. Political Propaganda in Antiquity. A Collection of Case Studies (Leiden, Boston 2005) 1-12 stellen ebenso wie die unreflektierte Verwendung des Begriffs, Propaganda' in den einzelnen Beiträgen einen Rückschritt dar. Vgl. zu neuen Perspektiven Rainer Gries, Zur Ästhetik und Architektur von Propagemen. Überlegungen zu einer Propagandageschichte als Kulturgeschichte, in: Ders., Wolfgang Schmale (Hg.), Kultur der Propaganda (Bochum 2005) 9-35.

${ }_{5}^{5}$ Aloys Winterling, Aula Caesaris. Studien zur Institutionalisierung des römischen Kaiserhofes in der Zeit von Augustus bis Commodus (31 v. Chr. - 192 n. Chr.) (München 1999) 117-160; Konrad Vössing, Mensa regia. Das Bankett beim hellenistischen König und beim römischen Kaiser (München u.a. 2004); Christophe Badel, L'audience chez les sénateurs, in: Jean-Pierre Caillet, Michel Sot (Hg.), L'audience. Rituels et cadres spatiaux dans l'antiquité et le haut moyen age (Paris 2007) 141-164. Hier nicht hinreichend berücksichtigt werden konnten Dirk Schnurbusch, Das Gastmahl römischer Aristokraten (Diss. Bielefeld 2005, unpubl., non vidi) und die gerade erschienene Studie von Fabian Goldbeck, Salutationes. Die Morgenbegrüßungen in Rom in der Republik und der frühen Kaiserzeit (Berlin 2010), der dem Aspekt der Interaktion und Kommunikation bei seiner Analyse einen prominenten Platz einräumt.
} 
nien oder die Triumphzüge ${ }^{6}$, sondern gerade die Totenfeiern boten Gelegenheit, retrospektiv den Rang des Verstorbenen und prospektiv denjenigen des neuen Kaisers in einem umfassenden Sinn vorzuführen und dabei das Publikum in einem ausgeklügelten performativen Akt zu integrieren.

Von besonderem Interesse ist die pompa funebris, die Septimius Severus im Jahr 193 aus machtpolitischem Kalkül zu Ehren des ermordeten und nach Ankunft des Severus konsekrierten Pertinax in Rom inszenierte und die der römische Senator sowie zweimalige Konsul Cassius Dio als Augenzeuge schildert ${ }^{7}$. Nach seinem prunkvollen adventus, den Dio in der panegyrischen Tradition des von Plinius überlieferten Einzugs Trajans schildert ${ }^{8}$, bereitete Septimius Severus sogleich die ApotheoseFeierlichkeiten für Pertinax vor. Die Errichtung eines Heiligtums, die Aufnahme des Divus Pertinax in die Eidesformeln, die spektakulär inszenierte Aufstellung eines goldenen Standbildes im Circus Maximus sowie dreier goldener Thronsessel in den Theatern ${ }^{9}$ wurden durch eine prunkvolle Bestattung abgerundet. Neben den Rostra wurde ein Holzgerüst errichtet, auf dem ein aus Elfenbein und Gold gearbeiteter Tempel mit einem aus den gleichen Materialien gearbeiteten Totenbett aufgestellt wurde, das ein wächsernes Abbild des verstorbenen Kaisers im Triumphalgewand, umhüllt von purpurnen und golddurchwirkten Decken trug. Der Kaiser und die Senatoren nahmen mit ihren Frauen auf dem Forum Platz, um den anschließenden Feierlichkeiten beizuwohnen. Am Totenbett wurden in feierlicher Prozession zunächst Bilder sämtlicher bedeutender Römer alter Zeit vorbeigetragen. Es folgten Chöre von Knaben und Männern, die Trauergesänge anstimmten. Darauf wurden bronzene Personifikationen der unterworfenen Völker gezeigt. Es schlossen sich Liktoren, Schreiber, Herolde und andere Gruppen kaiserlicher Bediensteter aus Rom an, denen wiederum Bildnisse bedeutender Personen folgten, die sich durch Taten, Entdeckungen oder ihre Lebensführung hervorgetan hatten. Nachdem auch die Reiter, Soldaten und die Rennpferde vorbeigezogen waren, wurden die Totenspenden vorgeführt, die der Kaiser, die Senatoren, angesehene Ritter, die Tribus und die städtischen Korporationen gestiftet hatten. Als Schlussakzent wurde ein vergoldeter, mit Elfenbein und indischen Edelsteinen verzierter Altar gezeigt. Septimius Severus hielt darauf die laudatio funebris, die von wehklagenden Akklamationen der Senatoren begleitet wurde. Die höchsten Priester und die amtierenden sowie für das Folgejahr designierten Magistrate hoben im Anschluss an die Rede das Totenbett an und übergaben es ausgewählten Rittern, die es auf das Marsfeld zum vorbereiteten ustrinum und dem rogus trugen, der ebenfalls mit Gold,

\footnotetext{
${ }^{6}$ Zum adventus siehe Joachim Lehnen, Adventus Principis. Untersuchungen zu Sinngehalt und Zeremoniell der Kaiserankunft in den Städten des Imperium Romanum (Frankfurt am Main 1997); vgl. auch die allgemeinen Beobachtungen bei Christian Ronning, Stadteinzüge in der römischen Republik. Die Zeremonie des ,adventus“ und ihre politische Bedeutung, in: Ders., Einblicke in die Antike. Orte - Praktiken - Strukturen (München 2006) 57-85. Zur Inszenierung im Rahmen des kaiserzeitlichen Triumphes siehe Ida Östenberg, Staging the World. Rome and the Other in the Triumphal Procession (Lund 2003); jetzt auch als dies., Staging the World. Spoils, Captives, and Representations in Roman Triumphal Procession (Oxford 2009); Mary Beard, The Roman Triumph (Cambridge, Mass. u.a. 2007). Für die Republik ferner Hans Beck, Züge in die Ewigkeit. Prozessionen durch das republikanische Rom, in: GFA 8 (2005) 73-104 sowie besonders Karl-Joachim Hölkeskamp, Hierarchie und Konsens. Pompae in der politischen Kultur der römischen Republik, in: Alexander H. Arweiler, Bardo M. Gauly (Hg.), Machtfragen. Zur kulturellen Repräsentation und Konstruktion von Macht in Antike, Mittelalter und Neuzeit (Stuttgart 2008) 79-126.

${ }^{7}$ Cass. Dio 75 (74), 4, 2-5, 5 mit Martin Zimmermann, Kaiser und Ereignis. Studien zum Geschichtswerk Herodians (München 1999) 312-314.

${ }^{8}$ Cass. Dio 75 (74), 1, 3-5. Der Abschnitt dürfte (wie 75 [74], 3, 1-3) von Cassius Dio aus seinem Frühwerk über Septimius Severus überommen worden sein und steht in deutlichem Gegensatz zur folgenden Episode (75 [74], 2, 1-6), in welcher der neue Kaiser scharf kritisiert wird. Siehe Zimmermann, Kaiser (wie Anm.7) 164.

${ }^{9}$ Cass. Dio 75 (74), 4,1 .
} 
Elfenbein, Standbildern und dem goldenen Wagen des Pertinax verziert war. Die Senatoren folgten dem Totenbett, wobei sich einige die Brust schlugen und andere Trauerlieder auf der Flöte spielten. Den Schluss des Zuges bildete der neue Kaiser. Das Wachsbild und die Totenopfer wurden auf den Scheiterhaufen gelegt. Während die Senatoren und der Kaiser auf hölzernen Tribünen Platz nahmen, veranstalteten die Magistrate, die jeweils nach Rang gekleideten equites, die übrige Reiterei und die Fußsoldaten die decursio. Die Konsuln entzündeten schließlich den Scheiterhaufen, worauf ein auffliegender Adler die Apotheose abschloss.

Um die Besonderheiten dieser Schilderung für die Frage nach dem hier repräsentierten kaiserlichen Rang würdigen zu können, sollte ein Blick auf die Bestattungsfeierlichkeiten geworfen werden, die keine zwanzig Jahre später für Septimius Severus selbst von seinen Söhnen Caracalla und Geta abgehalten worden sein sollen. Sie scheinen einem komplett gewandelten Zeremoniell gefolgt zu sein ${ }^{10}$. In der Schilderung Herodians begannen die Feierlichkeiten mit einer prunkvollen Aufbahrung am Kaiserpalast. Neben der Bahre mit dem Wachsbild trauerten auf der einen Seite der Senat und auf der anderen Seite Frauen, die bedeutende Männer oder Väter hatten. Nach sieben Tagen, an denen von Ärzten eine Verschlimmerung der Krankheit und schließlich der Tod des Kaisers inszeniert wurden, trugen die bedeutendsten equites gemeinsam mit jungen Männern des ordo senatorius die Bahre mit dem Wachsbild über die via sacra zu den Rostra. Auf Holztribünen sangen vornehme Kinder und die Frauen der Amtsträger Trauerlieder. Dann wurde die Bahre auf das Marsfeld zum ustrinum gebracht, wo ein reich mit golddurchwirkten Decken, Elfenbein und Gemälden geschmückter rogus errichtet wurde. Weihrauch, Räucherwerk, Früchte, Kräuter und Duftessenzen unterschiedlicher Art wurden auf den Scheiterhaufen gelegt. Alle Provinzen, Poleis und Amtsträger des Reiches hatten entsprechende Duftstoffe geschickt. Der ordo equester vollführte schließlich die decursio, wobei gleichzeitig Wagen mitfuhren, die Personen mit purpurgesäumten Kleidern und Masken trugen, die alle berühmten Feldherren und Kaiser wiedergaben. Der Kaiser zündete gemeinsam mit allen Übrigen den Scheiterhaufen an, und ein Adler entschwebte gen Himmel.

Die pompa auf dem Forum mit dem Defilee der Trauernden und die laudatio funebris scheinen neuartigen Ritualen im ustrinum zum Opfer gefallen zu sein, die auf eine gezielte Neugestaltung durch Caracalla und Geta zurückgeführt werden könnten. Dagegen spricht aber, dass Herodian seine Schilderung nicht als spezielle Zeremonie zu Ehren des Septimius Severus, sondern als verbindlichen Ablauf aller Apotheosefeiern versteht ${ }^{11}$. Um die Informationen beider Texte für die Rekonstruktion der römischen Apotheose heranziehen zu können, nahm man naheliegend an, dass die augenfälligen Unterschiede auf die persönliche Auswahl beider Autoren zurückzuführen seien, die Berichte folglich miteinander kombiniert werden können. Dies scheint sich auf den ersten Blick auch deshalb zu empfehlen, da der Originaltext Dios verloren und die Apotheose des Pertinax nur im Exzerpt des Geschichtswerkes erhalten ist, das der byzantinische Epitomator Xiphilinos im 11.Jahrhundert angefertigt hat. Paul Zanker etwa vermutete daher, dass Herodian zwar wichtige Einzelheiten nicht erwähnt, dafür aber wertvolle Informationen enthält, die im Exzerpt des Xiphilinos, aber auch bei anderen kaiserzeitlichen Autoren fehlen. Die unterschiedlichen Berichte ließen sich zu einem Gesamtbild zusammenfügen ${ }^{12}$.

\footnotetext{
${ }^{10}$ Herodian. 4, 2.

${ }^{11}$ Herodian. 4, 2, 1.

${ }^{12}$ Paul Zanker, Die Apotheose der römischen Kaiser (München 2004) 16 u.ö.
} 
Dieses Vorgehen ist angesichts des grundsätzlich problematischen Quellenwerts Herodians, der nicht einfach beiseite zu wischen ist, freilich unmöglich ${ }^{13}$. Die Fragwürdigkeit Herodians zeigt sich schon bei der angeblich siebentägigen Aufbahrung des Wachsbildes vor dem Kaiserpalast, in deren Rahmen aufwändig das Sterben des Kaisers inszeniert worden sein soll. Die Römer bahrten zwar ihre Toten im Atrium oder (wie im Fall des Augustus) im Vestibül auf ${ }^{14}$. Aber im Totenritual ging der Aufbahrung selbstverständlich die Feststellung des Todes voraus. Alle Elemente des Aufbahrungsrituals gehörten zum Totenkult, weshalb die Inszenierung eines über Tage sich hinziehenden Sterbevorgangs dem stadtrömischen Publikum kaum zuzumuten war, zumal man die aus Britannien nach Rom transportierte Urne mit der Asche des Septimius Severus bereits beigesetzt hatte. Auch die von Herodian behauptete Dauer der Aufbahrung von sieben Tagen ist sonst nirgends für römische Bestattungen belegt ${ }^{15}$. Doch auf die vielen Unstimmigkeiten seines Berichts kann an dieser Stelle nicht weiter eingegangen werden.

Was ist aber mit den unterschiedlichen Berichten anzufangen, wenn wir uns nicht darauf beschränken wollen, Cassius Dio als Augenzeugen zu loben und Herodian für seine Ungenauigkeiten zu tadeln? Wie können wir die Schilderungen nutzen, um das hier verfolgte Thema, nämlich die Repräsentation des kaiserlichen Ranges, zu erhellen?

Bevor wir die besondere Qualität beschreiben können, die der dionische Passus für die kaiserliche Repräsentation am Ende des 2. Jahrhunderts n. Chr. hat, muss zunächst der hohe Quellenwert des byzantinischen Exzerpts betont werden. Die Vorstellung nämlich, Xiphilinos habe einen sehr ausführlichen Bericht Dios in seiner Epitome entstellt und zusammengefasst ${ }^{16}$, ist irrig und trifft nicht den Charakter seiner Exzerpttechnik. Wo man den erhaltenen Originaltext mit dem Exzerpt vergleichen kann, wie etwa bei der Beschreibung der Bestattung des Augustus ${ }^{17}$, kann man feststellen, dass die Einzelheiten der pompa komplett abgeschrieben wurden und nur die laudationes fehlen. Wir dürften also auch bei der pompa des Pertinax im Exzerpt den Originaltext Dios ohne die von ihm erwähnte laudatio vor Augen haben ${ }^{18}$.

Was folglich beim Vergleich mit Herodian auffällt, ist, dass Dio offenbar weiß, wovon er spricht, während Herodian ein ihm und seinen Lesern in seiner politischen Symbolik weitgehend unverständliches Ritual beschreibt ${ }^{19}$. Cassius Dio verfolgte bewegt eine pompa funebris, die in ihrer Inszenierung dokumentierte, in welchem Umfang der Rang des Kaisers vom Zuspruch unterschiedlicher Bevölkerungsgruppen, nämlich der Senatoren, der Ritter, des Heeres, der stadtrömischen

${ }^{13}$ Herodian. 4, 2, 2. Auch die These, die eigentümliche Inszenierung des Ablebens habe Parallelen in der Herrschaftsübergabe neuzeitlicher Königshäuser, bei denen der neue Machthaber erst mit der Bestattung des Toten als neuer König installiert werden konnte, greift gerade für Rom nicht, da hier der neue Kaiser längst in vollem Umfang in Amt und Würden war.

${ }^{14} \mathrm{Zu}$ Augustus siehe Suet. Aug. 100; Cass. Dio 56, 34, 1f.

${ }^{15}$ Herodian ist die einzige antike Quelle, die eine Aufbahrung von sieben Tagen überliefert. Die in diesem Zusammenhang gern zitierten Parallelen bei Serv. Aen. 5, 64; 6, 218 (siehe etwa Joachim Engels, Funerum sepulcrorumque magnificentia [Stuttgart 1998] 177) sind wertlos, da sie auf falschen Annahmen beruhen (siehe bereits August Mau, Bestattung, RE 3, 1 [1897] 349).

${ }^{16}$ Zur Vermutung, Xiphilinos habe den Bericht Dios gekürzt, siehe Zanker, Apotheose (wie Anm. 12) z.B. 21 u.ö.

${ }^{17}$ Vgl. Ursul Ph. Boissevain (Hg.), Cassius Dio, Bd. 3 (Berlin 1955) 545-547 (Xiphilinos) mit ebd. Bd. 2 (Berlin 1955) 546f. (Dio).

${ }^{18}$ Sie dürfte im Original zwischen 75 (74), 5, 1 und 2 gestanden haben.

${ }^{19}$ Siehe die Einsichten bei Simon Price, From Noble Funerals to Divine Cult. The Consecration of Roman Emperors, in: David Cannadine, Simon Price (Hg.), Rituals of Royalty. Power and Ceremonial in Traditional Societies (Cambridge u.a. 1987) 56-105. 
Verwaltung, der stadtrömischen Korporationen und Tribus abhing. Als Bestattungsritual, das einer zeremoniellen Verständigung der Anwesenden über die politische wie gesellschaftliche Ordnung diente, gab die pompa Septimius Severus zugleich die Gelegenheit, sich als legitimer Erbe des Kaisers Pertinax zu präsentieren und damit zu signalisieren, dass seine eigene Machtstellung einen vergleichbaren $Z$ uspruch nicht nur verdiente, sondern voraussetzte.

Bemerkenswert an der Inszenierung ist aber noch etwas anderes: Was der aus Bithynien stammende Senator ohne eine Spur der Verwunderung beschreibt, ist eine Inszenierung der Stadt Rom als Hort alter Tradition und als Zentrum eines Reiches, dessen übrige Teile nicht vertreten sind, sondern bezeichnenderweise nur als Personifikationen unterworfener Völker vorgeführt werden ${ }^{20}$. Für den zweimaligen Konsul Cassius Dio war es selbst nach zweihundert Jahren Kaiserherrschaft eine Selbstverständlichkeit, dass Vertreter der Provinzen oder der Poleis in diesem Ritual keinen Platz hatten. Für den aus Kleinasien stammenden römischen Senator war Rom nicht nur Heimat, sondern der exklusive politische Bezugspunkt seiner sozialen Identität $t^{21}$. Entsprechend werden in seiner Darstellung der Kaiser, die ordines und die anderen Gruppen der stadtrömischen Gesellschaft in einem Bild zusammengefasst, als sei in zweihundert Jahren die Zeit stehen geblieben. Dies ist freilich nicht Absicht des Historiographen, sondern Kennzeichen des Bestattungsrituals selbst. Die Inszenierung von Kontinuität, Tradition und zeitloser Beständigkeit wird in der Feier trotz allen Wandels im zeremoniellen Ablauf ${ }^{22}$ nämlich akzentuiert, indem an der Spitze der pompa Bilder von „sämtlichen bedeutenden Römer(n) der alten Zeit“ vorangetragen werden ${ }^{23}$, womit sicherlich auch die summi viri der Republik gemeint sind. Die mediale Gestaltung steht folglich in der Tradition augusteischer Bildinventare, auf die man bei dieser Gelegenheit wie selbstverständlich zurückgriff2 ${ }^{24}$, zumal ein Kaiser wie Septimius Severus noch zu Beginn des 3.Jahrhunderts n. Chr. wie der Begründer des Prinzipats Augustus von sich sagen konnte, er habe nach den Bürgerkriegen die res publica wiederhergestellt ${ }^{25}$.

Für den Senator waren die traditionellen Formen des Rituals und die Verankerung kaiserlichen Ansehens bei den maßgeblichen Gruppen der stadtrömischen Bevölkerung entscheidend, er be-

${ }^{20}$ Price, Noble Funerals (wie Anm. 19) 85 betont die senatorische Tradition des Rituals. Zu dieser Romideologie siehe Martin Zimmermann, Rom - Zentrum und Spiegel der Welt, in: Jochen Johrendt, Romedio Schmitz-Esser (Hg.), Rom - Nabel der Welt. Macht, Glaube, Kultur von der Antike bis heute (Darmstadt 2010) 15-32.

${ }^{21}$ Siehe hierzu Géza Alföldy, Örtliche Schwerpunkte der medialen Repräsentation römischer Senatoren. Heimatliche Verwurzelung, Domizil in Rom, Verflechtung im Reich, in: Werner Eck, Matthäus Heil (Hg.), Senatores populi Romani. Realität und mediale Repräsentation einer Führungsschicht (Stuttgart 2005) 53-71, hier 56-60.

${ }^{22}$ Price, Noble Funerals (wie Anm. 19) 94-97 zur zunehmenden Bedeutung des Scheiterhaufens im Ablauf der Zeremonie.

${ }^{23}$ Cass. Dio 75 (74), 4, 5.

${ }^{24} \mathrm{Zu}$ den Bildnissen auf dem Augustusforum siehe Martin Spannagel, Exemplaria Principis. Untersuchungen zu Entstehung und Ausstattung des Augustusforums (Heidelberg 1999) 245-344; Uwe Walter, Memoria und res publica. Zur Geschichtskultur im republikanischen Rom (Frankfurt am Main 2004) 417-426; Tanja Itgenshorst, Augustus und die republikanische Tradition. Triumphalfasten und „summi viri“-Galerie als Instrumente der imperialen Machtsicherung, in: Hermes 132 (2004) 436-458.

${ }_{25}$ CIL VI 1033 (ob rem publicam restitutam). Siehe zum Hintergrund Alain M. Gowing, Empire and Memory. The Representation of the Roman Republic in Imperial Culture (Cambridge u.a. 2005) 150f. (zu Septimius Severus). Vgl. zur Kontextualisierung von Mitgliedern der Kaiserfamilie mit summi viri bzw. viri illustris ingeni auch die Kombination von Bildnissen des Germanicus und Drusus mit bedeutenden Schriftstellern und Rednern in der Tabula Hebana (Victor Ehrenberg, Arnold H.M. Jones, Documents Illustrating the Reigns of Augustus and Tiberius, Oxford ${ }^{2}$ 1976, Nr. 94a) Z.1-4 und Tac. ann. 2, 83. 
schwört den zweihundert Jahre alten consensus universorum ${ }^{26}$. Diese Exklusivität der Feier und die trotz einzelner jüngerer Elemente evidente Orientierung an den seit Beginn des Prinzipats entwickelten Formen herrscherlicher Repräsentation waren einem Mann wie Herodian, der fern der Hauptstadt lebte, unverständlich. Für ihn und seine Leser war daher die Vorstellung naheliegend, dass der rogus auf dem Marsfeld sich aus Duftstoffen zusammensetzte, die aus allen Teilen des Reiches zu Ehren des verstorbenen Kaisers entsandt wurden ${ }^{27}$. Beide Historiographen belegen trotz sehr unterschiedlicher Perspektive und trotz verschieden akzentuierter Schilderung aber das elementare Grundprinzip der Repräsentation kaiserlichen Ranges: Der in Form der beschriebenen Ehrungen artikulierte Zuspruch der ordines, gesellschaftlichen Gruppen, Korporationen oder Amtsträger definierte Rang und Ansehen des Kaisers ${ }^{28}$.

Die für die Herrschaftslegitimation des Septimius Severus grundlegende Inszenierung der Apotheose des Pertinax sollte daher nicht allein das Sozialprestige des Ermordeten verdeutlichen, sondern die beteiligten Personen gleichzeitig dem Nachfolger Septimius Severus verpflichten. Dies konnte nur gelingen, wenn die Inszenierung in ihren einzelnen Bestandteilen an bewährte Traditionen anknüpfte, die unter Augustus begründet worden waren und unter seinen Nachfolgern immer festere Formen angenommen hatten. Der Kaiser konnte zwar eine Inszenierung des bewährten Rituals vorschlagen, wichtiger war freilich die tatsächliche Mitwirkung der in das Ritual und die Zeremonie einbezogenen Personen, mithin der performative Akt selbst. Die an der zeremoniellen Ehrung des Pertinax Beteiligten forderten damit als politisch relevante Gruppen ihre Mitwirkung an der Herrschaft ein, während ihnen diese umgekehrt durch ihre aktive Beteiligung an der pompa symbolisch zugesichert wurde.

\section{Wahrnehmung und Anerkennung des kaiserlichen Ranges - die historische Dynamik seiner Repräsentation}

Die Repräsentation des Ranges funktionierte demnach nur, wenn er erkannt und anerkannt wurde. Aufrichtige Ehrungen waren die Belohnung für korrekt antizipierte und erfüllte Erwartungen. Die unter Augustus diskutierten und beschlossenen Ehrungen ${ }^{29}$, die bereits Beispiel überbordender Kaiserverehrung waren, markierten den Anfang einer langen Kette von Ehrenbeschlüssen, die Motor für die Modifizierung der herrscherlichen Repräsentation in den ersten beiden Jahrhunderten n. Chr. waren. Seit Beginn des Prinzipats war, wie Marianne Bergmann nachdrücklich betont, „das Überschießen der auf den Kaiser bezogenen Ehrungen (ein) essentieller Teil im Gefüge des Prinzipats ${ }^{\text {“ }}{ }^{30}$. Die Folge dieses „ständig ungeschriebene Gesetze überschreitenden Herrscherlobs“ sei die für den klassischen Archäologen bedenkliche Uneindeutigkeit der Bildsprache gewesen,

\footnotetext{
${ }^{26}$ Grundlegend Hans Ulrich Instinsky, Consensus universorum, in: Hermes 75 (1940) 265-278.

${ }^{27}$ Cass. Dio 75 (74), 4, 6 spricht davon, dass der Nachfolger, die Senatoren mit ihren Frauen, die führenden Ritter, die Tribus und die Korporationen der Stadt, also die an der pompa exklusiv beteiligte stadtrömische Bevölkerung, die Totenspenden bereit gestellt hatten.

${ }^{28}$ Siehe hierzu Lendon, Empire of Honour (wie Anm. 3) 120-129.

${ }^{29}$ Suet. Aug. 100.

${ }^{30}$ Bergmann, Strahlen (wie Anm. 2) 98.
} 
denn nicht ikonographische oder inhaltliche Stringenz des Dargestellten sei das Ziel gewesen, sondern die immer aufwändigere und gewohnte Bahnen verlassende Ehrung selbst ${ }^{31}$.

Von Interesse an diesen Beobachtungen ist für uns nicht in erster Linie die konstatierte ikonographische Unschärfe, die sich gleichermaßen in Texten feststellen ließe. Bedeutsamer ist, wie der Einfluss der Kaiser einerseits und jener der Führungsschichten sowie weiterer gesellschaftlichen Gruppen andererseits auf die Repräsentationsformen funktionierte. Welche Vorstellungen entwickelten demnach auf der einen Seite die Principes selbst von einer angemessenen Anerkennung ihrer Stellung? In welcher Form beeinflussten z.B. die Führungsschichten durch Antizipation, aufrichtige Verehrung, vorauseilenden Gehorsam und schlichte unaufrichtige Schmeichelei, mit welcher der persönliche Einfluss erhöht werden sollte, die Formen der Repräsentation und wie funktionierte die Verständigung hierüber in Dialog und Kommunikation?

Die Beobachtungen Bergmanns knüpfen an die grundlegenden Ausführungen Andreas Alföldis an, der immer wieder betont hat, dass die monarchische Repräsentation in ihren wesentlichen Elementen und in ihrem historischen Wandel auf Angebote der unterschiedlichen Gesellschaftsgruppen zurückzuführen ist ${ }^{32}$. Alföldi hat zwar vor allem interessiert, auf welchen Grundlagen die Tetrarchen und die spätantiken Kaiser bei der Gestaltung ihrer herrscherlichen Repräsentation aufbauten ${ }^{33}$, aber er untersuchte zu diesem Zweck nicht nur die Eigenarten spätantiker Repräsentation, sondern versuchte aufzuzeigen, dass ihre markante und oft für zeittypisch gehaltene Signatur vielmehr Wurzeln hat, die sich partiell bis in die Topik des klassischen Griechenlands zurückverfolgen lassen. Im Bild des griechischen Theaterkönigs etwa sah er das literarische Grundmuster der folgenden hellenistischen Herrschaftsrepräsentation angelegt, mit der sich die Römer intensiv auseinanderzusetzen hatten und von der sie eine Vielzahl von Elementen adaptierten $^{34}$. Dieser methodische Zugriff, bei dem partiell die spezifischen sozialen und politischen Kontexte ausgeblendet zu sein scheinen und die monarchische Repräsentation ein von der historischen Entwicklung gelöstes Eigenleben zu führen scheint, ist zwar verschiedentlich kritisiert worden $^{35}$, doch trifft diese Kritik nur partiell, denn Alföldi hatte einen durchaus treffenden Blick für die historischen Rahmenbedingungen des von ihm beschriebenen Wandel ${ }^{36}$. Für unser Thema ist etwa von Interesse, dass er die ersten beiden nachchristlichen Jahrhunderte in einer Zeitphase zusammenfassen zu können glaubte ${ }^{37}$. An verschiedenen Stellen seines Werkes hebt er hervor, dass in dieser Zeit im Rückgriff auf ältere Muster bereits in Grundzügen entworfen wurde, worauf dann im 3. und 4. Jahrhundert zurückgegriffen worden $\operatorname{sei}^{38}$. Vor allem dem Senat komme in den ersten beiden Jahrhunderten die maßgebliche Rolle bei der Entwicklung der kaiserlichen Repräsentation

\footnotetext{
${ }^{31}$ Ebd.

32 Alföldi, Repräsentation (wie Anm. 1) 32 zum Mechanismus, „wie sich diese Kriecherei unversehens festigte und als etwas Feststehendes von der Zentralgewalt übernommen, zu einer Vorschrift erhoben wurde“; 84.

${ }^{33}$ Ebd. IX-XVIII.

${ }^{34}$ Ebd. 9-25; Andreas Alfoldi, Gewaltherrscher und Theaterkönig, in: Kurt Weitzmann (Hg.), Late Classical and Medieval Studies in Honor of Albert M. Friend (Princeton 1955) 15-55.

${ }^{35}$ Siehe z.B. Winterling, Aula (wie Anm. 5) 31f.

${ }^{36}$ Siehe etwa auch die Studien Andreas Alfoldi, Die Geburt der kaiserlichen Bildsymbolik. Kleine Beiträge zu ihrer Entstehungsgeschichte, in: MH 7 (19509 1-13; MH 8 (19519 190-215; MH 9 (1952) 204-243; MH 10 (1953) 103-124; MH 11 (1954) 133-169.

${ }^{37}$ Andreas Alfoldi, Repräsentation (wie Anm. 1) 133: „...das Beibehalten und die Weiterentfaltung der hergebrachten Formen und das Absterben ihres alten Inhaltes sind eben die einander ergänzenden Merkmale des traianisch-hadrianisch-antoninischen Zeitalters".

${ }^{38}$ Ebd. 5 u.ö. Vgl. auch Frank Kolb, Herrscherideologie der Spätantike (Berlin 2001) 38-46.
} 
zu. Dies beruhe auf einer „sonderbaren Zwiespältigkeit der Stellung des Senats“: Sie sei durch „die formelle Zuerkennung der Vorherrschaft und den gleichzeitigen Verlust der Macht" gekennzeichnet gewesen ${ }^{39}$. Dieser Gegensatz habe das erste Jahrhundert bereits bestimmt, sei in aller Schärfe aber erst im 2. Jahrhundert zutage getreten ${ }^{40}$. Kaiser wie Senatoren hätten vor diesem Hintergrund, gewissermaßen dem background noise der Repräsentation, gemeinsam „neue Amtsabzeichen, neue Regeln der Etikette, neue Formen, die den neuen Aufbau einer immer mehr hierarchisch durchgegliederten Gesellschaft sichtbar machten, kreiert" ${ }^{\text {“1 }}$.

Sieht man sich die Repräsentation des kaiserlichen Ranges unter diesem Gesichtspunkt an, was hier nur in einigen wenigen Schlaglichtern geschehen kann, dann lassen sich zwei Grundelemente der herrscherlichen Repräsentation erkennen. Zum einen wollten die Kaiser, von den bekannten Ausnahmen einmal abgesehen, trotz der evidenten Ferne von der Menge der Beherrschten als Vertreter der gesamten Bürgerschaft erscheinen, mussten sich im Habitus also zumindest den führenden Schichten gegenüber, die ja eine entscheidende Kontaktzone darstellten, aufgeschlossen zeigen. Zum anderen konnten sie ihre tatsächliche Machtstellung nicht verhehlen und mussten für diese neue Stellung adäquate Repräsentationsformen finden, zumal beispielsweise die ordines im öffentlichen Auftreten äußerlich klar durch Rangabzeichen gekennzeichnet waren ${ }^{42}$. Marianne Bergmann hat in diesem Zusammenhang von „einer charakteristischen Spaltung der Repräsentationsformen“ gesprochen, die den Herrscher einerseits als Kaiser, andererseits als Magistraten republikanischer Tradition zeigten ${ }^{43}$. Um beide Aspekte verbinden zu können, schöpften die Kaiser im Bereich der äuBeren Erscheinung und der Insignien aus dem reichen Fundus republikanischer Herrschaftszeichen.

Doch zunächst zu den Formen des Habitus, die eine Nähe zur Bürgerschaft, insbesondere zum Senat und Ritterstand signalisierten ${ }^{44}$. Hierzu gehört zum Beispiel die Fortsetzung und Neugestaltung der salutationes, die in ihrer Ausweitung auf einen immer größer werdenden Personenkreis zugleich ihre persönlich verbindliche Form verloren ${ }^{45}$. Dennoch waren sie in einen weiteren Komplex des persönlichen Umgangs beispielsweise in Begrüßungsformen eingebunden, die in der öffentlichen Wahrnehmung von Bedeutung gewesen zu sein scheinen. Hierzu gehörten unter anderem der Wangenkuss, der mit Senatoren getauscht wurde, sowie die Darreichung der Hand zum Handkuss der Ritter ${ }^{46}$. Auch das Aufstehen gegenüber Senatoren war bis zur Zeit des Commodus üblich und wurde von Biographen sowie Historiographen ausdrücklich protokolliert ${ }^{47}$. Von besonderer Problematik war der Fußfall vor dem Kaiser ${ }^{48}$, denn er konnte als Proskynese missverstanden werden. Für Tiberius ist daher bezeugt, dass er diese schon in republikanischer Zeit üb-

\footnotetext{
${ }^{39}$ Alföldi, Repräsentation (wie Anm. 1) 131, vgl. auch $26 f$.

${ }^{40}$ Ebd. 54-56, 60f., 74 u.ö.

${ }^{41}$ Ebd. X.

${ }^{42}$ Siehe zuletzt den Überblick bei Scholz, Repräsentation (wie Anm. 1).

43 Bergmann, Repräsentation (wie Anm.2) 171; siehe auch Aloys Winterling, Caligula. Eine Biographie (München 2003) 118, der auch von einer „doppelbödigen Kommunikation, die sich mit dem Kaisertum in Rom etabliert hatte", spricht (142 u.ö.).

${ }^{44}$ Siehe zur falschen Nähe Alföldi, Repräsentation (wie Anm. 1) 25-29, 39, $100 \mathrm{f}$.

${ }^{45}$ Vgl. auch Winterling, Aula (wie Anm. 5) 117-144; Goldbeck, Salutationes (wie Anm. 5) passim.

${ }^{46}$ Alföldi, Repräsentation (wie Anm. 1) 40-42.

${ }^{47}$ Ebd. $42-45$.

${ }^{48} \mathrm{Zu}$ den republikanischen Wurzeln ebd. 49.
} 
liche Bittgeste grundsätzlich ablehnte ${ }^{49}$. Diese positive Haltung wurde akzentuiert, indem über das Einfordern des Fußkusses durch negative Herrscher wie Caligula oder Domitian berichtet wurde ${ }^{50}$.

Zum einfachen, schlichten Auftreten des Herrschers gehörte auch, dass er zu Fuß in der Stadt unterwegs war ${ }^{51}$. Eine Alternative war die Sänfte, die aber nach Möglichkeit - wie die Beispiele Augustus und Hadrian zeigen ${ }^{52}$ - geschlossen zu halten war, um die den Magistraten vorbehaltenen Ehrungen zu vermeiden. Auch wurde die Begegnung mit Magistraten und ihren Liktoren offenbar als problematisch empfunden ${ }^{53}$. Ein direktes Aufeinandertreffen wurde vermieden ${ }^{54}$. Diese Nachrichten beziehen sich auf eine eigentümliche Art der Repräsentation, die besonders darauf abzielte, die tatsächliche Stellung des Herrschers zu verschleiern. Der selbstverständlich sichtbare Kaiser wurde ostentativ unsichtbar gemacht ${ }^{55}$. Dieses Paradoxon machte den propagierten Inhalt, nämlich die herausragende Stellung, umso deutlicher. Dieses Phänomen begegnet auch in anderem Zusammenhang: Mit dem reisenden Herrscher wird die Tugend der celeritas kombiniert, hinter der sich von Beginn an auch der Versuch verbirgt, dem Kaiser in seiner außerordentlichen Stellung etwas Flüchtiges, Ungreifbares zu geben. Gleichzeitig wird sein persönliches Erscheinen mit einer Lichtmetaphorik verknüpft, die seine herausragende Stellung symbolisiert ${ }^{56}$. Er erscheint als strahlende Sonne, die alles Positive verkörpert, aber rasch vorbeizieht und somit wenig Belastung mit sich bringt.

In diesen Kontext performativer Akte, mit denen die bestehende Rangstellung negiert werden sollte, ist auch das Zugeständnis einzuordnen, neue Formen des öffentlichen Auftretens ebenfalls den Magistraten zuzugestehen. Im ersten Jahrhundert setzte sich beispielsweise der Tragstuhl bzw. die Sänfte als Transportmittel durch, welche seit Claudius den übrigen Magistraten als Fortbewegungsmittel gestattet wurde ${ }^{57}$. Ähnliches gilt für das Wagennutzungsrecht, das zunächst weiblichen Mitgliedern des Kaiserhauses, aber schließlich auch den obersten Magistraten zuerkannt wurde, so dass Ludwig Friedländer mit Blick auf das 3. Jahrhundert n. Chr. gar von einer „Kutschenaristokratie“ sprechen konnte ${ }^{58}$. Diese Art der Übertragung neuer Formen des öffentlichen Auftretens gilt ebenso für einzelne Insignien. So durften die Konsuln bei dem processus consula-

\footnotetext{
${ }^{49}$ Cass. Dio 57, 21, 7.

${ }^{50}$ Sen. benef. 2, 12, 1f.; Cass. Dio 59, 19, 5; 27, 1; Mart. lib. spect. 9, 66, 3; Plin. paneg. 24, 2; Alfoldi, Repräsentation (wie Anm. 1) 54f., 64.

${ }^{51}$ Ebd. 101.

52 Suet. Aug. 53, 2; Cass. Dio 69, 7, 2.

${ }^{53}$ Suet. Tib. 31, 2 .

${ }^{54}$ Alfolldi, Repräsentation (wie Anm. 1) $101 \mathrm{f}$.

${ }^{55}$ Siehe hierzu ausführlich Fritz-Heiner Mutschler, Potestatis nihilo amplius habui quam ceteri. Zum Problem der Invisibilisierung der Macht im frühen Prinzipat, in: Gert Melville (Hg.), Das Sichtbare und das Unsichtbare der Macht (Köln u.a. 2005) 259-282; Martin Jehne, Augustus in der Sänfte. Über die Invisibilisierung des Kaisers, seiner Macht und seiner Ohnmacht, in: Gert Melville (Hg.), Das Sichtbare und das Unsichtbare der Macht (Köln u.a. 2005) 283-307, der die geschlossene Sänfte als Versuch versteht, „Ehrerbietungsgesten, in denen die Sonderstellung des Augustus klar zutage trat“, zu vermeiden (290). Augustus habe „seine Präsenz in der Öffentlichkeit außerhalb der sorgsam choreographierten offiziellen Auftritte“ reduzieren wollen (306).

${ }^{56}$ Helmut Halfmann, Itinera Principum. Geschichte und Typologie der Kaiserreisen im Römischen Reich (Stuttgart 1986) 59f., 148-151; Michael Mause, Die Darstellung des Kaisers in der lateinischen Panegyrik (Stuttgart 1994) 192f.

${ }^{57}$ Suet. Claud. 28; App. civ. 3, 93; Cass. Dio 47, 10, 3 und 60, 2, 3. Alfoldi, Repräsentation (wie Anm. 1) $104 f$.

${ }^{58}$ Ebd. 106-108; Ludwig Friedländer, Darstellungen aus der Sittengeschichte Roms in der Zeit von Augustus bis zum Ausgang der Antonine, 4 Bde., 10. Aufl. hg. v. Georg Wissowa (Leipzig u.a. 1921-23) I 293; IV 22-25.
} 
ris sowie die Spielgeber bei den ludi weiterhin das Triumphalgewand zeigen ${ }^{59}$ und seit flavischer Zeit bei der prätorischen pompa, seit Trajan beim Konsulatsantritt ein Szepter tragen ${ }^{60}$.Zur Entwicklung der herrscherlichen Repräsentation gehörte also der nicht selbstverständliche Usus, dass Magistrate ihr Triumphalornat nach dem Vorbild des Kaisers durch neue Insignien erweitern konnten.

Die neuen Formen senatorischer Repräsentation, die in den letzten beiden Jahrzehnten intensiv erforscht wurden, zeigen jedoch deutlich, dass solche kalkuliert begrenzte Generosität von Seiten der Herrscher die faktische politische Entmachtung nicht verdecken konnte ${ }^{61}$. Man kann vielmehr davon sprechen, dass die Zunahme von Ehrentiteln und Statussymbolen Ergebnis und Folge der faktischen Entmachtung war. Frank Kolb hat daher zu Recht herausgestellt, dass die „auf die Person des Princeps als Repräsentanten des Staates zugeschnittene Hierarchie" und die damit einhergehende „Nivellierung der Bevölkerung im monarchischen Herrschaftssystem“ von einem „feingesponnenen System äußerlicher Differenzierung verschleiert" worden sei ${ }^{62}$.

Diese Mechanismen dürften den Mitgliedern der senatorischen Führungsschicht nicht verborgen geblieben sein. Der ordo lässt daher in seiner Repräsentation zwei Tendenzen erkennen, die der gewandelten Situation Rechnung tragen. Zum einen lassen sich in Folge der abnehmenden Chance, Konkurrenz öffentlich zu zeigen, Zeichen politischen Rückzugs erkennen. Thomas Schäfer etwa konnte am Beispiel der sella curulis und der fasces zeigen, dass in der Kaiserzeit eine „Verlagerung der magistratischen Selbstdarstellung in den Sepulkralbereich“ stattfand ${ }^{63}$. Und Henner von Hesberg verdeutlichte anhand der Sepulkralarchitektur und zuletzt noch einmal am Beispiel der senatorischen domus eine Introversion, die aus dem politischen Bedeutungsverlust der Senatoren resultierte ${ }^{64}$.

Neben dem Rückzug senatorischer Repräsentationsformen aus der Öffentlichkeit steht der Versuch, der neuen Stellung, die maßgeblich durch das Wohlwollen und die Gunst des Kaisers im Karriereverlauf beeinflusst wurde, adäquaten Ausdruck zu geben. Diese zweite neue Tendenz senatorischer Repräsentation wurde u.a. von Werner Eck und Géza Alföldy in allen Facetten beleuchtet ${ }^{65}$. Seit Augustus setzte sich ein neuer epigraphic habit durch, der sich durch die Abfassung ausführlicher Inschriften mit detaillierten Angaben zum cursus honorum auszeichnete. Dieser spiegelt

${ }^{59}$ Alfoldi, Repräsentation (wie Anm. 1) 93-100.

${ }^{60}$ Schaefer, Imperii Insignia (wie Anm. 2) 181-185.

${ }^{61}$ Alföldi, Repräsentation (wie Anm. 1) 121f. zur ,zweiten Art von kaiserlichen Abzeichen (...), die die monarchische Position unumwunden bezeichneten“.

${ }^{62}$ Kolb, Statussymbolik (wie Anm. 1) 250.

${ }^{63}$ Schaefer, Imperii Insignia (wie Anm. 2) 135-141.

${ }^{64}$ Henner von Hesberg, Die Häuser der Senatoren in Rom. Gesellschaftliche und politische Funktion, in: Eck, Heil, Senatores (wie Anm. 21) 19-52. Vgl. in diesem Zusammenhang und zur „,neuen Bedeutung der Innenräume und der Privatsphäre" auch Henning Wrede, Senatorische Sarkophage Roms. Der Beitrag des Senatorenstandes zur römischen Kunst der hohen und späten Kaiserzeit (Mainz 2001) bes. 57-66, 94-116, Zit. 114.

${ }^{65}$ Siehe z.B. jeweils mit weiteren Hinweisen Werner Eck, Senatorial Self-Representation. Developments in the Augustan Period, in: Fergus Millar, Erich Segal (Hg.), Caesar Augustus. Seven Aspects (Oxford 1984) 129-167; Werner Eck, Roma Caput Mundi - the Eternal City as Monument and Idea. The Elite of the Empire in the Public Space of the Capital Rome (Wellington 2001); ders., „Tituli Honorarii“, curriculum vitae und Selbstdarstellung in der Hohen Kaiserzeit, in: Acta colloquii epigraphici Latini Helsingiae 1991 (Helsinki 1995) 211-237; ders., Der Senator und die Öffentlichkeit - oder: Wie beeindruckt man das Publikum?, in: Eck, Heil, Senatores (wie Anm. 21) 1-18; Géza Alföldy, „Pietas immobilis erga principum“ und ihr Lohn. Öffentliche Ehrenmonumente von Senatoren in Rom während der Frühen und Hohen Kaiserzeit, in: Ders., Silvio Panciera (Hg.), Inschriftliche Denkmäler als Medien der Selbstdarstellung (Stuttgart 2001) 11-46; Alföldy, Schwerpunkte (wie Anm. 21) $53-72$. 
zum einen die neuartige Hierarchisierung der Führungsschichten wider ${ }^{66}$ und sollte zum anderen ungeachtet der zunehmenden Differenzierung die Nähe zum jeweiligen Kaiser dokumentieren. Die Amtsträger wurden so in die neue Ordnung eingebunden, was schon an den Statuen, die Augustus von Senatoren auf dem Augustusforum aufstellen ließ, sinnfällig wurde. So konnten die Senatoren auch in den gewandelten Verhältnissen ihr lebhaftes Interesse an der Repräsentation ihrer Stellung befriedigen, obwohl der Kaiser sie zunehmend aus dem öffentlichen Raum der Stadt Rom selbst verdrängte. Die Nekropolen am Rande der Stadt und die Privatgrundstücke der prächtigen Villen traten als Repräsentationsräume an die Stelle der öffentlichen Plätze Roms ${ }^{67}$.

Damit kommen wir zum zweiten Aspekt herrscherlicher Inszenierung, nämlich zur Frage einer adäquaten Darstellung des höchsten Ranges und zu den Mitteln, mit denen Distinktion verdeutlicht wurde. Es ist selbstverständlich, dass der Kaiser als princeps generis humani mit der ihm eigenen Machtfülle und religiösen Aura als Stellvertreter Jupiters auf der Erde unbestrittenes Haupt der res publica war. Am kaiserlichen Rang gab es keinerlei Zweifel und dennoch stellte sich die grundsätzliche Frage, wie dieser Rang in Rom (darauf müssen wir uns beschränken) in äußeren Formen zu repräsentieren war, ohne die Fiktion einer res publica restituta aufgeben zu müssen.

Trotz aller Zurückhaltung bei öffentlichen Auftritten und der Repräsentation von Unsichtbarkeit, von der antike Autoren bei positiven Herrschern gern berichten, war immer klar, wer in einer Gruppe öffentlich auftretender, führender Männer der Kaiser war ${ }^{68}$. Die ihn begleitenden Liktoren, zunächst zwölf, seit Domitian vierundzwanzig und die Prätorianer boten bereits ein eindrucksvolles Schauspiel. Die vorangetragenen Fackeln und Feuerbecken, zu Ehren des Kaisers vorgetragene Lieder und Akklamationen, besondere Sitze im Senat und bei den Spielen wie die exklusiv von ihm getragenen ornamenta hoben den Princeps sichtbar von den Senatoren und den primores des Ritterstandes $a^{69}$. Die Entourage, die den Herrscher ständig begleitete, ließ auf den Straßen Roms aber auch auf den römischen Straßen und in den Provinzstädten keinen Zweifel, wer personaler Kern des Zuges war ${ }^{70}$.

Das Janusgesicht herrscherlicher Repräsentation, nämlich die Leugnung und Akzentuierung seiner außerordentlichen Stellung, tritt besonders deutlich in seiner Kleidung zutage, die ständig zwischen beiden Polen wechselte und der jeweils übernommenen Rolle als Konsul, Princeps, Triumphator, Priester usw. angepasst wurde ${ }^{71}$. Bereits im ersten Jahrhundert legten die Kaiser in Rom, sicherlich aber auch auf Reisen penibel Wert darauf, dass ihre Kleidung der jeweiligen

${ }^{66}$ Peter Eich, Zur Metamorphose des politischen Systems in der römischen Kaiserzeit (Berlin 2005) 20-45; Werner Eck, Auf der Suche nach Personen und Persönlichkeiten. Cursus honorum und Biographie, in: Konrad Vössing (Hg.), Biographie und Prosopographie. Internationales Kolloquium zum 65. Geburtstag von Antony Birley (Stuttgart 2005) 53-72; Karl-Joachim Hölkeskamp, Herrschaft, Verwaltung und Verwandtes. Prolegomena zu Konzepten und Kategorien, in: Rudolf Haensch, Johannes Heinrichs (Hg.), Herrschen und Verwalten. Der Alltag der römischen Administration in der Hohen Kaiserzeit (Köln u.a. 2007) 1-18, hier 8-10. Vgl. auch zum Spannungsverhältnis Hierarchisierung/gesellschaftliche Schichtung und soziale Gruppen sowie zur gegenseitigen Bedingtheit von „politischer Ordnung und gesellschaftlicher Stratifikation“: Aloys Winterling, ,Staat', ,Gesellschaft' und politische Integration in der römischen Kaiserzeit, in: Klio 83 (2001) 93-112, hier 109.

${ }^{67}$ Alföldy, Repräsentation (wie Anm. 1).

${ }^{68}$ Ebd. 128.

${ }^{69}$ Ebd. 140-186.

${ }^{70}$ Halfmann, Itinera Principum (wie Anm. 56) 90-110, 117-124. Siehe zur Inszenierung des Herrschers im Gefolge z.B. Tac. hist. 2, 59. Vgl. Alföldi, Repräsentation (wie Anm. 1) 128.

${ }^{71}$ Siehe nur Christian Ronning, Herrscherpanegyrik unter Trajan und Konstantin. Studien zur symbolischen Kommunikation in der römischen Kaiserzeit (Tübingen 2007) 106-115. 
Situation angemessen war $^{72}$. Auf der einen Seite sehen wir die konsequente Entwicklung eines kaiserlichen Prachtgewandes. Standardgewand war zwar die senatorische toga praetexta, aber seit Claudius sind inschriftlich verschiedene Personen am Hof überliefert, die sich um die aufwändigen kaiserlichen Kleidungsstücke zu kümmern hatten ${ }^{73}$. Wir hören von der regia vestis sowie der vestis alba triumphalis, zwei Kleidungsstücken, die mit den Triumphalinsignien exklusiv vom Kaiser als Festkleider getragen wurden. Ferner gab es die vestis castrensis, zu der auch das purpurne paludamentum gehörte, das im Laufe der ersten beiden Jahrhunderte geradezu zum Sinnbild des kaiserlichen Imperators und der Kaiserherrschaft überhaupt wurde. Commodus etwa verstand sich als Sohn Mark Aurels daher als porphyrogennetos ${ }^{74}$. Zu festlichen Anlässen wurde spätestens seit Caligula, Claudius und Nero von den Kaisern selbst sowie ihren Frauen purpurne Kleidung mit Goldstickereien angelegt ${ }^{75}$. Bei Commodus bildeten goldverziertes paludamentum und tunica die ständig getragenen Kleidungsstücke ${ }^{76}$. Auch purpurne Hosen, die bracae, wurden seit Trajan Bestandteil des kaiserlichen Militärkostüms, das im 2. Jahrhundert immer häufiger von den Kaisern in Rom getragen wurde ${ }^{77}$. In unbekanntem Umfang dürften am Ende des 2.Jahrhunderts dann bereits auch edelsteinverzierte Bestandteile des Ornats regelmäßig vorgekommen $\operatorname{sein}^{78}$. Die allmählich aufkommenden, aber über die zwei Jahrhunderte immer aufwändiger gestalteten Festkostüme und Galakleidung, die die Kaiser bei allen bedeutenden öffentlichen Auftritten anlegten, fanden ihre Entsprechung in der Gestaltung von Tragsitz, sella curulis, Wagen und Zaumzeug der Pferde. All dies wird vergoldet, mit Elfenbein und Edelsteinen verziert, um es deutlich von den entsprechenden Insignien der Magistrate oder den ihnen zugestandenen Wagen und Tragsitzen abzuheben $^{79}$. Cassius Dio berichtet, dass auf dem Scheiterhaufen des Pertinax auch der goldene Wagen verbrannt wurde, mit dem er sich durch die Stadt bewegt hatte ${ }^{80}$. Die eingangs erwähnten prachtvollen Gegenstände, die bei der pompa funebris gezeigt wurden, entsprachen also jenen Insignien und Prachtmöbeln, welche die Kaiser auch zu Lebzeiten benutzten.

Wir können also sehen, dass die kaiserliche Kleidung von Beginn an in äußerst luxuriösen Varianten gefertigt wurde. Dies ist freilich nur eine Seite, denn mit einer täglich wohl mehrmaligen mutatio vestis versuchten die Kaiser - auch im Sinne einer Negierung ihrer Stellung - zurückhaltende Auftritte ${ }^{81}$. So gab es neben den Prachtgewändern Abteilungen für Kleider, welche Nähe zu den Beherrschten symbolisieren sollten ${ }^{82}$. Die Abteilung der vestis imperatoria privata war vermutlich der einfachen Kleidung vorbehalten, während die forensis vestis die etwa bei der salutatio getragene Toga meint. Von Interesse für die Sorgfalt bei der Auswahl der jeweils passenden Kleidung sind die ebenfalls von Freigelassenen geführten Abteilungen der vestis scaenica et gladiatoria, vestis venatoria

${ }^{72}$ Zur mutatio vestis siehe Alfoldi, Repräsentation (wie Anm. 1) 123-127; Zimmermann, Kaiser (wie Anm.7) $222-232$.

${ }^{73}$ Siehe auch Winterling, Aula (wie Anm. 5) 99f. mit Einzelnachweisen.

${ }^{74}$ Herodian. 1, 5, 5 .

${ }^{75}$ Alfoldi, Repräsentation (wie Anm. 1) 144-146, 176 mit Verweis auf Suet. Calig. 19, 2; Cass. Dio 59, 17, 3 (für Caligula), Plin. nat. hist. 33, 3, 63; Tac. ann. 12, 56 (zu Claudius) und Suet. Nero 25, 1 (zu Nero).

${ }^{76}$ Cass. Dio 73 (72), 17, 3; Hist. Aug. Comm. 8, 8.

${ }_{77}$ Alföldi, Repräsentation (wie Anm. 1) 178-180.

78 Siehe hierzu die Severertondo mit den Kaiserbildnissen im Juwelenschmuck bei Heinrich Heinen, Herrscherkult im römischen Ägypten und die „damnatio memoriae“ Getas, in: RM 98 (1991) 263-298.

${ }^{79}$ Alföldi, Repräsentation (wie Anm. 1) 106-110, 150-160.

${ }^{80} \mathrm{Vgl}$. Anm. 8 .

${ }^{81}$ Kolb, Herrscherideologie (wie Anm. 38) 54.

${ }^{82}$ Siehe einzelne Belege bei Winterling, Aula (wie Anm. 5) $99 \mathrm{f}$. 
und vestis munda. Aufmerksamkeit verdient ferner die Garderobe der vestis Graecula. Für Tiberius, Claudius, Domitian und Hadrian ist belegt, dass sie im Rahmen von Agonen anstelle der römischen Toga das vielleicht regelmäßig purpur gefärbte griechische Himation getragen haben ${ }^{83}$.

Wenn Nero also nach seiner Griechenlandtournee in purpurner Kleidung und mit einer mit goldenen Sternen verzierten Chlamys bekleidet in Rom einzieht, ist er nur auffälliges Glied einer längeren Kette, die schließlich in die exzentrischen Auftritte des Commodus mit seidenem Untergewand und ähnlich gearbeiteter purpurner Chlamys mündet ${ }^{84}$. Prachtgewänder und situationsbezogene Kostüme standen allen Kaisern zur Verfügung. Die kritischen Berichte Suetons über Neros Auftritte und jene Dios über Commodus verweisen auf das grundlegende Problem, mit Hilfe der antiken Überlieferung die Repräsentation des kaiserlichen Ranges angemessen zu rekonstruieren. Andreas Alföldi hat mit Blick auf diese Überlieferung von der „Umklammerung des rhetorischen Schlinggewächses“ gesprochen, die der „Rekonstruktion der sehr langen und allmählichen Entwicklung" im Weg stehe ${ }^{85}$. Literarische Topoi und die ständig wiederholten Vorwürfe einer am hellenistischen Königtum orientierten, unrömischen Ausgestaltung der Repräsentation erschweren in der Tat, ihre Entwicklung wertfrei und in ihrer tatsächlichen Gestaltung wie Zielsetzung $\mathrm{zu}$ rekonstruieren und einzuordnen. Es ist bezeichnend, dass Neuerungen in der Gestaltung des monarchischen Auftretens grundsätzlich ausführlich nur bei mali principes gewürdigt werden. Sie werden dabei regelmäßig auf Initiativen dieser Kaiser zurückgeführt, und ihre Akzeptanz bei den Zeitgenossen wird weitgehend geleugnet.

An verschiedenen Beispielen wurde mittlerweile in der neueren Forschung verdeutlicht, dass gerade von den mali principes Standards geschaffen wurden, die von ihren Widersachern und Nachfolgern übernommen und weitergeführt wurden. Die Doppelgesichtigkeit der kaiserlichen Repräsentation ist auch gerade darin begründet, dass offiziell übernommene und schließlich eingeforderte Formen der Ehrung neben immer neuen Varianten, die von den unterschiedlichen Gruppen der Gesellschaft ersonnen wurden, standen. Die Entwicklung beispielsweise des Senatorenstandes zeigt dies: Die von seinen Mitgliedern gewählten Formen der Lebensführung und Standesrepräsentation kultivierten abgekoppelt vom Kaiserhof Formen, die man einem Kaiser gleichzeitig, zumindest vorläufig, noch absprach. Dies gilt etwa für die verzerrte Wahrnehmung und Darstellung des neronischen Kaiserpalastes ${ }^{86}$, aber auch für andere Formen von Entgleisungen, die angeprangert werden. Ein Thrasea Paetus etwa kann als Tragöde auftreten und zugleich Nero solche Ambitionen vorwerfen ${ }^{87}$. Vespasian kann Pläne Neros, die Hauptstadt nach Alexandria zu verlegen, kritisieren und gleichzeitig in Ägypten entwickelte Repräsentationsformen dieses Kaisers nach Rom tragen ${ }^{88}$. Aus dieser Diskrepanz zwischen eigener Haltung und Kritik an anderen sollte

${ }^{83}$ Suet. Tib. 13, 1 (Tiberius); Cass. Dio 60, 6, 1f. (Claudius); Suet. Dom. 4, 4 (Domitian); Cass. Dio 69, 16, 1 (Hadrian).

${ }^{84}$ Suet. Nero 25 (Nero); Cass. Dio 73 (72), 17, 3 (Commodus).

${ }^{85}$ Alföldi, Repräsentation (wie Anm. 1) 4f., 19, 23 (zum „merkwürdigen Eigenleben der Rhetorik“), 176 (zum „Motivzwang der rhetorischen Invektive“).

${ }^{86}$ Marianne Bergmann, Der Koloss Neros, die Domus Aurea und der Mentalitätswandel im Rom der frühen Kaiserzeit (Mainz 1994).

${ }^{87}$ Zum Kontext siehe Christian Ronning, Der Konflikt zwischen Kaiser Nero und P. Clodius Thrasea Paetus. Rituelle Strategien in der Frühen Römischen Kaiserzeit, in: Chiron 36 (2006) 329-355.

${ }^{88}$ Martin Zimmermann, Der Kaiser als Nil. Zur Kontinuität und Diskontinuität von Repräsentation im Frühen Prinzipat, in: Gregor Weber, Martin Zimmermann (Hg.), Propaganda - Selbstdarstellung - Repräsentation im römischen Kaiserreich des 1.Jhs. n. Chr. (Stuttgart 2003) 317-348. Vgl. auch zu den Strategien der flavischen Herrschaftsrepräsentation ders., Die restitutio honorum Galbas, in: Historia 54 (1995) 56-82. Zum 
man freilich nicht automatisch eine Ehrenrettung eines Kaisers wie Nero ableiten, der in seinen Handlungen und den gewählten Formen der Selbstdarstellung durchaus von einer „bestürzenden und zugleich naiven Egozentrik" geleitet gewesen sein mag ${ }^{89}$.

Die Verehrung des Kaisers nahm auf unterschiedlichen Ebenen sehr verschiedene Formen an, die in großem Umfang auch irrational, d.h. jenseits aller offiziellen ideologischen Begründungszusammenhänge gestaltet wurden. Solche Spuren finden sich angefangen von der domus Augusta bis hin zu den einzelnen Gemeinwesen des Reiches. Förderlich für diese Entwicklung war zweifellos, dass eine zentral gesteuerte Propaganda kaiserlicher Repräsentation nicht existierte ${ }^{90}$. Die verbindlichen Standards waren derart vage und uneingeschränkt positiv gefasst, dass sie problemlos Konsens fanden. Der Herrscher stand einfach für Wohlergehen, Glück und Blüte des Reiches wie seiner einzelnen Teile. Solche Formen der Repräsentation konnte man als Appellativa ohne Bedenken übernehmen. Bei den Formen der lokal ersonnenen Ehrungen konnten die Kaiser in Kommunikation mit örtlichen Amtsträgern und Magistraten umgekehrt überlegen, was in das offiziell abgesteckte Programm aufzunehmen war und überregionales Interesse versprach.

Ansonsten scheint die Programmatik spätestens im 2.Jahrhundert in den immer gleichen Formeln und Schlagworten erstarrt zu sein. Dies lag schon deshalb nahe, da man seit Augustus mit dem Kaiser nicht nur das Goldene Zeitalter assoziierte, sondern diesem Zustand eine vom aktuellen Herrscher gelöste, überzeitliche Dauer unterstellte. Der Kaiser als rector orbis wählte daher den Himmelsglobus als „Hauptmotiv der Kaisersymbolik ${ }^{\text {“91 }}$. Er verband symbolisch die zeitlich begrenzte Herrschaft mit der ewigen kosmischen Ordnung ${ }^{92}$. Seit den Flaviern wurde verstärkt die aeternitas der Kaiser artikuliert und mit Schlagworten wie abundantia, concordia, felicitas, laetitia, providentia, securitas usw. kombiniert, die nicht beliebig erweiterbar waren ${ }^{93}$. Man darf davon ausgehen, dass die Kommunikation über solche Tugenden und ihre Auswahl recht unprätentiös verlief. Die Vorstellung, dass der Kaiser gewissermaßen laufend unter Stress stand, da er seine Herrschaftsberechtigung gegenüber den verschiedenen gesellschaftlichen Gruppen unter Beweis stellen zu müssen glaubte, ist sicherlich anachronistisch ${ }^{94}$.

Wie wir aber auch sahen, sollte man sich von dieser Firnis erstarrter Repräsentationsformen nicht täuschen lassen. Daneben wurde laufend Neues erprobt und adaptiert. Zu erwähnen ist etwa die von Peter Weiss jüngst ingeniös für Antoninus Pius und Mark Aurel nachgewiesene Verpflichtung römischer Eheleute, in Rom vor einem Standbild des jeweiligen Kaiserpaares zu opfern, nachdem die

Weiterleben Domitians als Gott und Stammvater von Göttern in der Dichtung nach der damnatio memoriae siehe die Zeugnisse bei J. Rufus Fears, The Cult of Jupiter and Roman Imperial Ideology, ANRW II 17.1 (1981) 3-141, hier 80; Jens Leberl, Domitian und die Dichter. Poesie als Medium der Herrschaftsdarstellung (Göttingen 2004).

${ }^{89}$ Siehe zu einem für diese Frage wichtigen Text Marianne Bergmann, Hatte Nero ein politisches und/oder kulturelles Programm? Zur Inschrift von Akraiphia, in: Jean-Michel Croiselle, Yves Perrin (Hg.), Neronia VI. Rome à l'époque néronienne (Brüssel 2002) 273-284, hier 281.

${ }^{90}$ Zimmermann, Nil (wie Anm. 88) 11-40.

${ }^{91}$ Alfoldi, Repräsentation (wie Anm.1) 235; Rolf M. Schneider, Roma Aeterna - Aurea Roma. Der Himmelsglobus als Zeitzeichen und Machtsymbol, in: Kodikas/Code. Ars Semeiotica 20 (1997) 103-132.

${ }^{92}$ Siehe Ronning, Herrscherpanegyrik (wie Anm. 71) 110f. zum Kaiser als „emblematischer Figur, in der sich alle Bürger und der Staat selbst aufgehoben finden".

${ }^{93}$ Siehe bereits Paul L. Strack, Untersuchungen zur römischen Reichsprägung des zweiten Jahrhunderts, Bd. 1 (Stuttgart 1931) 187; Alföldi, Repräsentation (wie Anm. 1) 201f.; Martin P. Charlesworth, Providentia and aeternitas, in: HThR 29 (1936) 107-132.

${ }^{94}$ Siehe hierzu Armin Eich, Politische Literatur in der römischen Gesellschaft. Studien zum Verhältnis von politischer und literarischer Öffentlichkeit in der späten Republik und frühen Kaiserzeit (Köln u.a. 2000) $360 f$. 
kaiserliche Ehe in aufwändigen Inszenierungen als Sinnbild der concordia gefeiert und zum Vorbild für die Ehe schlechthin stilisiert worden war $^{95}$. Auch die weitergehende Entmachtung des Senats bei gleichzeitig zunehmender Anzahl der Abbildungen des Genius Senatus auf Münzen und öffentlichen Denkmälern gehört ebenfalls in diesen Kontext einer schleichenden Weiterentwicklung herrscherlicher Repräsentation ${ }^{96}$. Hinter ihr stehen die Aushöhlung alter Inhalte und ein fortschreitender gesellschaftlicher Wandel. Die bei der pompa funebris des Pertinax repräsentierte politische Ordnung war selbstverständlich nicht mehr jene der flavischen Zeit, auch wenn sie sich so gab. Und wenn Dio Septimius Severus einen adventus trajanischer Zeit zuschreibt, dann war ihm, wie dem Geschichtswerk an vielen Stellen zu entnehmen ist, sehr wohl bewusst, dass dies nurmehr Fassade herrscherlicher Repräsentation war, der er in rhetorischer Tradition stehende Worte lieh. Ansonsten können wir feststellen, dass die Schaffung neuer Repräsentationsformen des Kaisers und die gleichzeitige Inszenierung einer gesellschaftlichen Hierarchie mittels Standesabzeichen, Insignien und Ehrenrechten durchschlagend etabliert werden konnte. Dies lässt sich an der unrechtmäßigen Adaptation der Statussymbole ${ }^{97}$ sowie an schon beispielsweise von Martial oder Petron verfassten satirischen literarischen Darstellungen eines Habitus studieren, mit dem bestehende gesellschaftliche Standesgrenzen überschritten werden sollten. Auch der Versuch von Mitgliedern der Führungsschicht, durch die Initiative zur Publikation kaiserlicher Dokumente eigenes Ansehen zu erwerben, gehört in diesen Kontext ${ }^{98}$.

Zusammenfassend lassen sich folgende Strukturelemente in der Repräsentation kaiserlichen Ranges erkennen:

1. Ein wesentliches Element herrscherlicher Repräsentation war die topische Behauptung, der Kaiser unterscheide sich in Aussehen und Auftreten nicht von anderen Magistraten. Er benutze dieselben Insignien und falle im Stadtbild nicht als ranghöchste Person auf.

2. Trotz evidenten gesellschaftlichen Wandels und einer völligen Veränderung der Machtverhältnisse wurde das strukturelle Weiterbestehen einer überkommenen Ordnung behauptet, die Grundlage der Repräsentation war.

3. Die tatsächliche Machtstellung brachte es freilich mit sich, dass der weitgehend entmachtete Senat, der zunehmend bedeutende Ritterstand und andere gesellschaftliche Gruppen spezielle Sonderrechte und bis dahin unbekannte Ehrungen vorschlugen, die zu einer allmählichen Veränderung der Repräsentation führten.

4. Parallel hierzu erweiterten die Kaiser den öffentlich zur Schau gestellten Prunk, der nun auch Ornat und Herrschaftsinsignien erfasste, die immer prachtvoller und aufwändiger gestaltet wurden. Gleichzeitig hielt die von der Führungsschicht getragene Biographie und Historiographie aber zum Großteil daran fest, diesen Umstand zu leugnen.

\footnotetext{
95 Siehe das hochinteressante Beispiel bei Peter Weiss, Die vorbildliche Kaiserehe. Zwei Senatsbeschlüsse beim Tod der älteren und der jüngeren Faustina, neue Paradigmen und die Herausbildung des, antonischen Prinzipats, in: Chiron 38 (2008) 1-45.

${ }^{96}$ Alföldi, Repräsentation (wie Anm. 1) 134f.; Zimmermann, Galba (wie Anm. 88) hier 73-75 (zur Einführung des Genius auf Münzabbildungen durch Galba und die Adaptation der Programmatik durch Vespasian); vgl. auch Jean Béranger, Der Genius populi Romani in der Kaiserpolitik, in: BJ 154 (1965) 72-85.

${ }_{97}^{97}$ Reinhold Meyer, Usurpation of Status and Status Symbols, in: Historia 20 (1971) 275-302.

${ }^{98}$ Werner Eck, Kap. Administrative Dokumente. Publikation und Mittel der Selbstdarstellung, in: Ders., Die Verwaltung des Römischen Reiches in der Hohen Kaiserzeit, Bd. 2 (Basel 1997) 359-382, hier 376-380; Rudolf Haensch, Einführung, in: Ders. (Hg.), Selbstdarstellung und Kommunikation. Die Veröffentlichung staatlicher Urkunden auf Stein und Bronze in der römischen Welt (München 2009) $11 \mathrm{f}$.
} 
5. Auf Seiten der Senatoren, Ritter und lokalen Magistrate wurden neue Formen der Repräsentation entwickelt, die als Rahmen der herrscherlichen Repräsentation fungierten, aber unter diesem Aspekt noch nicht untersucht worden sind. Dies kann als Forschungsdesiderat nur angedeutet werden.

6. Der Rang war grundsätzlich auch dadurch zu repräsentieren, dass der Kaiser das höchste Ansehen genoss. Alles Positive wurde auf ihn zurückgeführt, alle persönlichen Verdienste Einzelner ihm gutgeschrieben. Allein dieses grundlegende Element kaiserlicher Repräsentation machte Herrschaft immer prekär.

\section{Neue Forschungsfelder: Anwesenheitskultur, Habitus, Dynamik der Repräsentation und die notwendige Revision des ,Akzeptanzsystems}

Nachdem im vorhergehenden Teil verschiedene Realia der Repräsentation vorgestellt wurden, soll abschließend auf einer abstrakteren Ebene betrachtet werden, zwischen welchen Polen gesellschaftlicher Aushandlungsprozesse sich die Repräsentation des kaiserlichen Ranges bewegt und welche Forschungsfelder sich daraus ergeben haben und zukünftig als Perspektive wissenschaftlicher Arbeit anbieten. Verschiedentlich ist angeklungen, dass die Grundprinzipien der Repräsentation nur zu verstehen sind, wenn man sie als Bestandteil eines komplizierten Kommunikationsprozesses versteht. Die Beobachtung, die Gerd Althoff für das Mittelalter formuliert hat, dass „Rangdenken ... ganz offensichtlich eine Kultur der Repräsentation im Sinne von Zur-Schau-Stellung“ hervorgebracht habe ${ }^{99}$, lässt sich auf die römische Kaiserzeit übertragen. Die römische Gesellschaft war stark von der öffentlichen Darstellung und Wahrnehmung des jeweiligen gesellschaftlichen Ranges geprägt ${ }^{100}$. Öffentlichkeit und sozialer Status waren eng aufeinander bezogen, denn kaiserliches wie senatorisches Handeln bedurfte traditionell der Sichtbarkeit ${ }^{101}$. Erst die Augenzeugenschaft der Anwesenden und die Teilnahme an einem symbolisch-rituellen Akt, wie der eingangs beschriebenen Bestattungszeremonie, beglaubigten die abstrakten und teilweise fiktiven Bestandteile der Ordnung. Dies gilt auch für den täglichen Umgang mit Amtsträgern oder Senat. In einer solchen für vormoderne Gesellschaften typischen Präsenzkultur ${ }^{102}$ versicherten sich die Beteiligten in physi-

${ }^{99}$ Gerd Althoff, Die Kultur der Zeichen und Symbole, in: Ders., Inszenierte Herrschaft. Geschichtsschreibung und politisches Handeln im Mittelalter (Darmstadt 2003) 274-297, hier 277.

${ }^{100}$ Siehe z.B. Hanns Gabelmann, Antike Audienz- und Tribunalszenen (Darmstadt 1984) 117-177; Ronning, Stadteinzüge (wie Anm. 6) 57-61.

${ }^{101}$ Siehe zur für Senatoren zunehmend begrenzten Öffentlichkeit, die nun der Kaiser einnimmt, auch Peter Eich, Aristokratie und Monarchie im kaiserzeitlichen Rom, in: Hans Beck u.a. (Hg.), Die Macht der Wenigen. Aristokratische Herrschaftspraxis, Kommunikation und ,edler ' Lebensstil in Antike und Früher Neuzeit (München 2008)145-151 (mit weiteren Hinweisen). Vgl. auch die Überlegungen zur Rolle der „visibility“ in antiken Monarchien von Olivier Hekster, Richard Fowler, Introduction, in: Dies. (Hg.), Imaginary Kings. Royal Images in the Ancient Near East, Greece and Rome (Stuttgart 2005) 9-38 und für die römische Kaiserzeit Olivier Hekster, Captured in the Gaze of Power. Visibility, Games and Roman Imperial Representation, in: ebd. $157-176$.

${ }^{102}$ Zum aus der Kultursoziologie entlehnten Begriff der Präsenzkultur siehe Barbara Stollberg-Rilinger, Des Kaisers alte Kleider. Verfassungsgeschichte und Symbolsprache des Alten Reiches (München 2008) 9-12, 299305. Vgl. dies., Einleitung, in: Dies. (Hg.), Vormoderne politische Verfahren (Berlin 2001) 9-24. Siehe ferner grundlegend Rudolf Schlögl, Vergesellschaftung unter Anwesenden. Zur kommunikativen Form des Politischen 
scher Anwesenheit, dass sie die Ordnung unterstützten und davon ausgingen, dass alle anderen die gleichen Erwartungen mit dieser Ordnung verbanden wie sie selbst. Auch verständigte man sich darüber, dass die Maxime des politischen Handelns nicht Abstrakta waren, sondern in der Praxis umgesetzt wurden ${ }^{103}$.

Deshalb wurde das, was in der Öffentlichkeit vorgeführt wurde, für wahr gehalten. Dies gilt in besonderem Maße für eine Herrschaftsordnung wie den Prinzipat, dessen fiktiven Bestandteile, nämlich die ostentativen Anleihen aus der Zeit der Republik, grundsätzlich fraglich waren. Daher war diese Herrschaftsordnung besonders darauf angewiesen, dass die Beteiligten laufend versicherten, diese Konstruktion trotz ihres allzu offensichtlich fiktiven Charakters zu akzeptieren und zu unterstützen. Die weiterhin bestehende Geltung der republikanischen Traditionen und historisch gewachsenen Identität musste folglich in unterschiedlichen Kontexten und Signaturen unter gewandelten Verhältnissen betont werden ${ }^{104}$. Das historisch Einzigartige bei der Konstituierung des Prinzipats war der Umstand, dass die über Jahrhunderte gewachsenen habituellen Konzepte und Formen symbolischer Kommunikation mit der Entstehung des Prinzipats grundsätzlich zur Disposition standen. Dies umso mehr, da, wie wir oben gesehen haben, in einer völlig neuartigen politischen Architektur die Repräsentation des kaiserlichen Ranges auf der Fiktion beruhte, dass die hierfür bereit stehenden Mittel sich nicht von denen unterschieden, die auch anderen Mitgliedern der Führungsschicht zur Verfügung standen. Mit dem öffentlich inszenierten Verzicht auf die Demonstration der kaiserlichen Stellung beabsichtigten die Kaiser, ihre Machtstellung zu festigen und auszubauen. Diese paradoxe Konstruktion funktionierte angesichts der tatsächlichen Machtfülle, die allen Beteiligten bekannt war, aber nur, solange die Adressaten im wörtlichen Sinn mitspielten und diesen Teil der Inszenierung als einen Teil der Wirklichkeit akzeptierten. Diese Akzeptanz war insbesondere im Bereich der Invisibilisierung der neuen Machtstellung folglich stets prekär. Das öffentliche Agieren des Kaisers und der an der Macht Beteiligten wurde schon von den antiken Protagonisten als ein Schauspiel verstanden ${ }^{105}$, in dem die aristokratischen Akteure mit den ihnen darin zugewiesenen Rollen oft haderten ${ }^{106}$.

in der vormodernen Stadt, in: Ders. (Hg.), Interaktion und Herrschaft. Die Politik der frühneuzeitlichen Stadt (Konstanz 2004) 9-60.

${ }^{103}$ Eich, Literatur (wie Anm. 94) 353f. Mit Blick auf die „Repräsentation staatlicher Macht“ stellt er fest, dass die Kaiser ihrer „hoheitlichen Gewalt“ eine „wahrnehmbare Gestalt“ gegeben hätten: „Diese Gestaltgebung vollzog sich primär in der Herrschaftspraxis, indem der Herrscher in bestimmten Formen agierte und andere in die Formen ritualisierten Agierens mit einbezog." Diese Herrschaftspraxis sei in Bildern und Texten reproduziert worden.

${ }^{104}$ Zur „Geltung“ siehe Gerhard Schönrich, Ulrich Baltzer, Die Geltung von Geltungsgeschichten, in: Gert Melville, Hans Vorländer (Hg.), Geltungsgeschichten. Über die Stabilisierung und Legitimierung institutioneller Ordnungen (Köln u.a. 2002) 1-26 und die übrigen Beiträge in dem Band.

${ }^{105}$ Siehe Suet. Aug. 100.

106 Siehe z.B. Cass. Dio 73 (72), 4, 2f.; 75 (74), 2, 1-3. Vgl. Alföldi, Repräsentation (wie Anm. 1) 123 („Tagesordnung des Kaisers“ als „wahres Theater“). Im Extremfall wurden das Bühnenspiel selbst und die Reaktionen des Publikums auf Auftritte von Kaisern und Mitgliedern der Führungsschicht zunehmend politisch aufgeladen und es einstand ein spezieller Erprobungsraum kaiserlicher Repräsentation wie der Publikumsreaktionen auf diese Versuche der Auslotung neuer Darstellungsmuster. Siehe z.B. die Überlegungen von Shadi Bartsch, Actors in the Audience. Theatricality and Doublespeak from Nero to Hadrian (Cambridge, Mass. 1994); Nicholas Purcell, Does Caesar Mime?, in: Bettina Bergmann, Christine Kondoleon (Hg.), The Art of Ancient Spectacle (New Haven u.a. 1999) 181-194; das Kapitel „Extreme Mimesis. Spectacle in the Empire", in: Anne E. Duncan, Performance and Identity in the Classical World (Cambridge u.a. 2006) 188-217 
Insbesondere unter den Mitgliedern der führenden Schichten gab es folgerichtig einen lebhaften formellen wie informellen Austausch darüber, wie der persönliche Habitus der jeweiligen Stellung adäquat gestaltet, im Laufe einer Karriere sowie eines damit verbundenen sozialen Aufstiegs eventuell angepasst oder als Folge tektonischer Schwankungen im Gesamtgefüge gar grundsätzlich neu definiert werden sollte ${ }^{107}$. Das dahinter stehende öffentliche Bewusstsein für habituelle Konformität lässt sich schon in der Republik als provokative Aufhebung dieser auf Konsens angewiesenen Ordnung studieren ${ }^{108}$. Zur Inszenierung der (senatorischen) Person wie des kaiserlichen Ranges gehören neben Insignien beispielsweise auch die Bewegung (Gehen, Fahren, Schreiten usw.), das Sprechen (Ansprachen, Gespräche usw.) sowie Gestik und Mimik ${ }^{109}$. Die Erwartungen, welche die Zeitgenossen mit einer solchen habituellen Inszenierung von sozialem Rang und Herrschaft verbanden, waren offenbar verbindlich, denn ihre Nichterfüllung zog Befremden bis hin zur Aufkündigung politischer Gefolgschaft nach sich, wie etwa die Beispiele Claudius ${ }^{110}$ oder Commodus zeigen ${ }^{111}$. In der immer präsenten Sensibilität dafür, ob ein Verhalten angemessen war oder nicht, lag auch oder gerade in der Kaiserzeit die besondere historische Dynamik der auf Öffentlichkeit aufbauenden Kommunikation: Unstimmigkeiten wurden rasch wahrgenommen, hohler Schein entlarvt und entsprechende Änderungen in Symbolinventar, Handlungsabläufen, Choreographie, Dramaturgie und beteiligtem Personal angemahnt.

Die Integration des Körpers ${ }^{12}$ und seiner Inszenierung in die Kommunikation über die Rangstellung des Kaisers barg also gewisse Gefahren, hatte aber auch große Vorteile. Im Gegensatz $\mathrm{zu}$ schriftlichen und mündlichen Mitteilungen, deren Inhalt nicht einfach zurückgenommen werden konnte, konnte mit dem Körper indirekt kommuniziert werden. Rudolf Schlögl hat daher zu Recht hervorgehoben, dass der Körper „Schutz vor der Zwangsläufigkeit interaktiver Kommunikation“ und die „Möglichkeit von Kommunikation auf Widerruf " bot ${ }^{113}$. Bei der recht schwierigen Formierung der kaiserlichen Rangstellung und bei der tastenden Ausbildung ihrer

und Martin Zimmermann, Extreme Formen physischer Gewalt in der antiken Überlieferung, in: Ders. (Hg.), Extreme Formen von Gewalt in Bild und Text des Altertums (München 2009) 155-192, hier 183-189.

${ }^{107}$ Für die mediale Umsetzung siehe bereits Richard Brilliant, Gesture and Rank in Roman Art - the Use of Gesture to Denote Status in Roman Sculpture and Coinage (New Haven 1963); Jutta Ronke, Magistratische Repräsentation im römischen Relief. Studien zu standes- und statusbezeichnenden Szenen (Oxford 1985), den Überblick bei Scholz, Repräsentation (wie Anm.1) 409-431 sowie jetzt auch Angelika Starbatty, Aussehen ist Ansichtssache. Zur kommunikativen Funktion von Kleidung in der Antike (Diss. München 2009). Zur Konstitution des senatorischen Ansehens siehe allgemein Christophe Badel, La noblesse de l'empire romain. Les masques et la vertu (Champ Vallon 2005).

${ }^{108}$ Siehe Tonio Hölscher, Provokation und Transgression als politischer Habitus in der späten Republik, in: RM 111 (2004) 83-104.

${ }^{109}$ Siehe bereits Carl Sittl, Die Gebärden der Griechen und Römer (Leipzig 1890, ND 1970) hier 78-173; Egon Flaig, Ritualisierte Politik. Zeichen, Gesten und Herrschaft im Alten Rom (Göttingen 2003) 99-122 u.ö.; Antony Corbeill, Nature Embodied. Gesture in Ancient Rome (Princeton 2004). Eine Untersuchung zur Rolle des kaiserlichen und senatorischen Körpers steht noch aus. Vgl. für die Frühe Neuzeit die Beiträge zum „Körper als Medium“ in dem Sammelband Johannes Burkhardt, Christine Werkstetter (Hg.), Kommunikation und Medien in der Frühen Neuzeit (München 2005) 429-460.

${ }^{110}$ Siehe z.B. Dieter Timpe, Claudius und die kaiserliche Rolle, in: Volker M. Strocka, Die Regierungszeit des Kaisers Claudius (41-54 n. Chr.). Umbruch oder Episode (Mainz 1994) 35-42.

${ }^{111}$ Zimmermann, Kaiser (wie Anm. 7) 59-63 mit Dio 73 (72), 4, 2 (Xiph.).

${ }^{112}$ Siehe hierzu etwa Jon Hall, The Deference-Greeting in Roman Society, in: Maia 50 (1998) 413-426.

${ }^{113}$ Rudolf Schlögl, Typen und Grenzen der Körperkommunikation in der Frühen Neuzeit, in: Burkhardt, Werkstetter, Kommunikation (wie Anm. 109) 547-560, hier 550. Vgl. André Kieserling, Kommunikation unter Anwesenden. Studien über Interaktionssysteme (Frankfurt am Main 1999) 147-178. 
Repräsentation hatte eine solche Möglichkeit, die kommunizierten Inhalte auf ihre Wirkung hin zu prüfen, den Vorteil, nicht akzeptierte Signale schlicht bestreiten zu können. Die Gegenseite hatte umgekehrt die Option, sie zu ignorieren oder eben zu bekräftigen.

Die Kategorie des Habitus ist also im Anschluss an Pierre Bourdieu umfassend zu verstehen: Der Begriff beinhaltet unterschiedliche Aspekte der persönlichen Erscheinung, zu denen alle relevanten Aspekte des sozialen Handelns bis hin zur bloßen physischen Präsenz gehören ${ }^{114}$. Der Habitus ist erkennbar an erlernten und unbewusst eingesetzten Handlungen, die zur historisch klar konturierten sozialen und politischen Stellung gehören und in sehr verschiedenen, bereits durch die republikanische Aristokratie entwickelten und nun neu definierten politischen, sozialen und religiösen Rollenmustern sowie den damit verbundenen Erwartungen aufgehen ${ }^{115}$. Nach Bourdieu ist der Habitus die „wirkende Präsenz der gesamten Vergangenheit, die ihn erzeugt hat " ${ }^{116}$. Für ihn ist er ein „System dauerhafter und übertragbarer Dispositionen“, die als „Erzeugungs- und Ordnungsgrundlage für Praktiken und Vorstellungen "117 dienten, dabei aber in einer „Spontaneität ohne Wissen und Bewusstsein "118 wirkten. Dies schließt die Möglichkeit ein, immer neue Verhaltensweisen hervorzubringen, die auf die Erfordernisse einer gegenwärtigen und möglicherweise gewandelten sozialen wie politischen Situation reagieren.

Den Rahmen für die habituelle Kommunikation bildeten die vielfältigen Formen von Begegnung in Zeremonie, Ritual und politischen Alltagsgeschäften ${ }^{119}$. Hier fand der Kaiser die adäquaten Gelegenheiten, in denen sein überragender Rang wie in einem Brennspiegel präsentiert werden konnte. Anders als in der Spätantike, als die außerordentliche kaiserliche Stellung unterstrichen wurde, indem man ihn buchstäblich visuell und räumlich entrückte ${ }^{120}$, konstituierte sich die kai-

${ }^{114}$ Die verschiedentlich erwähnten äußerlichen Zeichen, die insignia imperii, begründen nicht die kaiserliche Stellung. Vgl. Tac. hist. 3, 68 (zum gescheiterten Versuch des Vitellius, die insignia im Concordia-Tempel niederzulegen). Nur der Tod, die komplette physische Auslöschung der Person, konnte die Stellung aufheben. Siehe zur Kategorie Bourdieus Beate Krais, Gunter Gebauer, Habitus (Bielefeld 2002).

${ }^{115}$ Für die Republik siehe Hans Beck, Die Rollen der Adeligen. Prominenz und aristokratische Herrschaft in der römischen Republik, in: Ders. u.a. (Hg.), Die Macht der Wenigen. Aristokratische Herrschaftspraxis, Kommunikation und ,edler' Lebensstil in Antike und Früher Neuzeit (München 2008) 101-123, der vor allem die Systemtheorie Niklas Luhmanns (Soziale Systeme. Grundriß einer allgemeinen Theorie [Darmstadt 2002]) fruchtbar machen möchte, in der „soziales Rollenverhalten“ als "partieller Habitus“ verstanden wird; vgl. Uwe Walter, Aristokratische Existenz in der Antike und der Frühen Neuzeit - einige unabgeschlossene Überlegungen, in: Hans Beck u.a. (Hg.), Die Macht der Wenigen. Aristokratische Herrschaftspraxis, Kommunikation und ,edler' Lebensstil in Antike und Früher Neuzeit (München 2008) 367-394, 375; siehe zur Kaiserzeit Ronning, Herrscherpanegyrik (wie Anm.71) 106-129.

${ }^{116}$ Krais, Gebauer, Habitus (wie Anm. 114) 6.

${ }^{117}$ Pierre Bourdieu, Sozialer Sinn (Frankfurt am Main 1987) 98.

118 Ebd. 105.

${ }^{119}$ Zur Bedeutung von Ritualen und Zeremonien siehe Barbara Stollberg-Rilinger, Zeremoniell, Ritual, Symbol. Neue Forschungen zur symbolischen Kommunikation in Spätmittelalter und Früher Neuzeit, in: Zeitschrift für historische Forschung 27 (2000) 389-405; Gerrit J. Schenk, Zeremoniell und Politik. Herrschereinzüge im spätmittelalterlichen Reich (Köln 2003); Jürgen Martschukat, Steffen Patzold (Hg.), Geschichtswissenschaft und „performative turn“. Ritual, Inszenierung und Performanz vom Mittelalter bis zur Neuzeit (Köln 2003) und für das antike Rom siehe Richard C. Beacham, Spectacle Entertainments of Early Imperial Rome (New Haven u.a. 1999); Stéphane Benoist, Rome, le prince et la Cité. Pouvoir impériale et cérémonies publiques ( ${ }^{\text {er }}$ siècle av.-début du IVe siècle apr. J.-C.) (Paris 2005); Hölkeskamp, Hierarchie (wie Anm. 6).

${ }^{120}$ Otto Treitinger, Die oströmische Kaiser- und Reichsidee nach der Gestaltung im höfischen Zeremoniell (Darmstadt ${ }^{2}$ 1956) 52 f.; Alfolddi, Repräsentation (wie Anm. 1) 38; Kolb, Herrscherideologie (wie Anm. 38) 117-125, bes. 121 . 
serliche Rangstellung in der frühen und hohen Kaiserzeit vornehmlich durch seine Handlungen in der Öffentlichkeit. Performanz und Herrschaftsausübung fielen demnach in der Person des Kaisers zusammen. Um in der Aktion, im Ritual und in den Handlungsabläufen Botschaften übermitteln zu können, mit denen die vom Publikum erwarteten und individuell von den Herrschern bevorzugten Qualitäten des Kaisers angezeigt wurden, waren die Handlungen zu einem gewissen Teil in standardisierte Muster festzuschreiben. Ihr repetitiver Charakter erleichterte die Wahrnehmung der Qualitäten, die propagiert wurden. Die Muster legten den dahinter stehenden Sinn fest, blieb aber in gewissem Umfang variabel und anpassungsfähig. Auch in Abweichungen, Erweiterungen und Improvisationen verloren diese fest gefügten Handlungsmuster nicht an Wirkung: Die Wahrnehmbarkeit der Abwandlung, Weiterentwicklung und Akzentuierung einzelner Elemente ist vielmehr Zeichen der ihnen inne wohnenden Dynamik.

Es ging bei diesen im 1. und 2.Jahrhundert immer weiter entwickelten und zunehmend differenzierten performativen Akten wohlgemerkt nicht in erster Linie darum, die Machtfülle zu verbergen, sondern konkret um Herrschaftsausübung in einem affirmativen, bisweilen aber auch konstitutiven Sinne. Armin Eich etwa nennt dies „formgebende Vergegenwärtigung von Herrschaft “121. Die Stellung des Kaisers selbst war dabei, wie Tonio Hölscher betont hat, durch die „Polarität von faktischer Macht und ideeller Herrschaft “122 gekennzeichnet. Der Herrscher musste demnach seine Macht und Überlegenheit tatsächlich durchsetzen, ausüben und erhalten. Zugleich war der Princeps aber auch verpflichtet, dieses Handeln als gerecht, fürsorglich usw. darzustellen.

Diese ideelle Seite der Machtausübung musste medial in Zeit und Raum vermittelt werden ${ }^{123}$. Dies ist in einem umfassenden Sinne zu verstehen: Die Räume, in denen sich der Kaiser bewegte, waren in ihrer Wahrnehmung und Gestaltung vielfältig kulturell kodiert ${ }^{124}$. Sie waren der durch Architektur, Bilder, Musik, Gerüche und anderes mehr gestaltete Rahmen, in dem sich die Personen zu unterschiedlichen, oft zeremoniell festgelegten Zeitpunkten bewegten. Die Topographie der Stadt Rom war in ihrer symbolischen Ordnung bekannt, so dass die darin vollzogenen Handlungen des Herrschers für Beteiligte wie Zuschauer verständlich waren ${ }^{125}$. Die Stadt war demnach die Bühne dieses Schauspiels, bei dem die Herrschaft des Kaisers ihren unmittelbaren Ausdruck fand.

${ }^{121}$ Eich, Literatur (wie Anm. 94) 355.

${ }^{122}$ Tonio Hölscher, Macht, Raum und visuelle Wirkung. Auftritte römischer Kaiser in der Staatsarchitektur von Rom, in: Joseph Maran u.a. (Hg.), Konstruktion der Macht. Architektur, Ideologie und soziales Handeln (Hamburg u.a. 2006) 185-201, hier 186. Siehe in diesem Zusammenhang die Überlegungen Paul Zankers, Prinzipat und Herrscherbild, in: Gymnasium 86 (1979) 353-368.

${ }^{123}$ Hölscher, Macht (wie Anm.122) 185-201; vgl. für das Mittelalter und die Frühe Neuzeit z.B. den Sammelband Werner Paravicini (Hg.), Zeremoniell und Raum. 4. Symposium der Residenzen-Kommission der Akademie der Wissenschaften Göttingen (Sigmaringen 1997) sowie Stéphane Benoist, L'espace urbain de Rome, comme lieu d'encadrement de la foule au premier siècle de l'Empire, in: Alain Leménorel (Hg.), La rue, lieu de sociabilité? Rencontres de la rue (Rouen 1997) 215-223.

${ }^{124}$ Zur sozial-politischen Valenz des Raumes siehe Martin Zimmermann, Stadtraum, Architektur und öffentliches Leben in der hellenistischen Stadt, in: Ders., Albrecht Matthaei (Hg.), Stadtbilder im Hellenismus (München 2009) 23-40, hier 23-26.

${ }^{125}$ Die unterschiedlichen städtischen Räume konnten daher auch Ort besonderer, auf den spezifischen Raum hin orientierten Ehrungen sein. Siehe hierzu etwa die Studie zu verschiedenen Ehrungskonzepten von Alexander Heinemann, Eine Archäologie des Störfalls. Die toten Söhne des Kaisers in der Öffentlichkeit des frühen Prinzipats, in: Fernande und Tonio Hölscher (Hg.), Römische Bilderwelten. Von der Wirklichkeit zum Bild und zurück (Heidelberg 2007) 41-109. Siehe in diesem Zusammenhang auch die Überlegungen von Badel, L'audience (wie Anm. 5) zur „logique spatiale“ bei den salutationes. 
Von den tatsächlichen Handlungen und standardisierten Abläufen im politischen Alltagsgeschäft haben wir meist keine konkreten Vorstellungen, aber die Topik historiographischer Beschreibungen und die repetitiven Bildmuster der Staatsreliefs signalisieren, wie man sich einen bestimmten Teil dieser öffentlichen Akte vorzustellen hat. Die in den Staatsreliefs etwa der profectio, adlocutio oder adventus-Szenen suggerierte Übersichtlichkeit des Rituals soll die dahinter ablaufende feingliedrige Maschinerie politischer und administrativer Vorgänge verstellen, denn Einsicht in ihren Ablauf hätte die Wahrnehmung des Rituals gestört und somit die mit dem Schauspiel beabsichtigte Vermittlung konkreter und oft schlichter Inhalte verhindert und nahezu unmöglich werden lassen. Inszenierung und Choreographie waren jedenfalls durch Verdichtung des Handlungsgerüstes und Eingrenzung der beteiligten Personen oder die Wiedergabe von Personifikationen darauf ausgerichtet, dass die intendierten Inhalte gesehen und im Ablauf erkannt werden konnten. Vor diesem Hintergrund verwundert es nicht, dass die Abbildung des Kaisers im Alltag der Herrschaft denselben Simplifizierungen, Konzentrationen und thematischen Begrenzungen folgte ${ }^{126}$. Ziel auch dieser Bilder und Texte ist die Suggestion gelungener Herrschaftspraxis, nicht die authentische Wiedergabe des Alltagsgeschäfts. Bilddetails, die den Alltag wiedergaben, sollten der allegorisierenden Szenerie nur den Anschein von Authentizität verleihen.

Ein besonders wichtiger Aspekt in einer Kommunikation, die auf Anwesenheit ausgerichtet ist, ist die Abbildung und Kommentierung von Herrschaftsrepräsentation in Bild und Text selbst. Dieser Aspekt ist daher so bedeutsam, da in der medialen Wiedergabe die Einbeziehung der Abwesenden in die kommunikative Konstellation ihren Platz hat. Sie erhalten durch die Beschreibung der skizzierten Vorgänge in Literatur, Historiographie und Reliefs die Möglichkeit, selbst mittels Betrachtung und Reflexion präsent zu sein ${ }^{127}$. Hier gibt sich zugleich die Chance, durch Rezeption, aber auch Textproduktion in die Herrschaftsrepräsentation einbezogen zu werden. Daher können insbesondere Texte auch Medien sein, um auf vielfältige Weise den Verlust politischer Partizipation einzuklagen oder mittels der Artikulation von Erwartungshorizonten umzusetzen $^{128}$. Von besonderer Bedeutung ist die mediale Präsentation, da sie im realen Vollzug umgesetzte Kommunikationsmuster wiedergeben, kommentieren, negieren, aber ebenso durch alternative Muster ersetzen kann. Die darin begründete inhärente Widersprüchlichkeit der Quellen ist in dieser Hinsicht bei Weitem nicht hinreichend erforscht. Welche diskursiven Verknüpfungen in der Antike zwischen öffentlichen Auftritten, Bildern, Texten und ideologischen Konzeptionen bestanden, ist bisher allenfalls in Ansätzen nachzuzeichnen. Es ist außerordentlich schwierig, die Formen und Mittel der symbolischen Kommunikation näher zu beschreiben, die sich zwischen dem Kaiser und einzelnen Personen sowie Gruppen entwickelt hatte.

Es ist viel darüber nachgedacht worden, was letztlich die Stabilität des Systems bewirkte. Dabei wird im Sinne des Beschriebenen seit geraumer Zeit betont, dass die Rangstellung des Kaisers wie überhaupt sein Machterhalt weniger auf Legalität, Gewalt und militärischer Durchsetzungskraft,

${ }^{126}$ Henner von Hesberg, Der Alltag des Kaisers nach der Bildüberlieferung auf Denkmälern der trajanischen Zeit - Ideologie, mediale Bedingungen und Realität, in: Rudolf Haensch, Joachim Heinrichs (Hg.), Herrschen und Verwalten. Der Alltag der römischen Administration in der Hohen Kaiserzeit (Köln u.a. 2007) 19-30, hier 29.

${ }^{127}$ Hans-Ulrich Gumbrecht, Diesseits der Hermeneutik. Die Produktion von Präsenz (Frankfurt am Main 2004) 99-110.

${ }^{128}$ Siehe zu diesem Aspekt kaiserzeitlicher Literatur Martin Zimmermann, Enkomion und Historiographie. Entwicklungslinien der kaiserzeitlichen Geschichtsschreibung vom 1. bis zum frühen 3. Jahrhundert n. Chr., in: Ders. (Hg.), Geschichtsschreibung und politischer Wandel im 3. Jahrhundert n. Chr. (Stuttgart 1999) 17-56. 
sondern auf Kommunikation mit seinen Zeitgenossen beruhte ${ }^{129}$. Insbesondere die von Egon Flaig vorgetragene These, die Herrschaft und Macht habe wesentlich auf Akzeptanz bei Heer, Senat und plebs urbana beruht, findet zur Zeit überraschend viele Anhänger, da sie der Kategorie der charismatischen Herrschaft Max Webers sehr nahe kommt und vorzüglich mit dem kommunikativen Aspekt kaiserlicher Repräsentation korrespondiert ${ }^{130}$. Verdienstvoll an diesen Überlegungen ist zweifellos der Hinweis, dass die Stellung des Kaisers der Unterstützung und Zustimmung bedurfte und hierfür entsprechende Strategien eingeschlagen werden mussten. Dennoch ist die Teilung der Adressaten in die genannten drei Gruppen viel zu schematisch, da die Stratigraphie der römischen Gesellschaft und auch der stadtrömischen Bevölkerung weitaus komplizierter war und die personellen Überschneidungen zwischen den Gruppen die politische Kommunikation prägten ${ }^{131}$.

Die verschiedentlich in antiken Quellen unterschiedlicher Art begegnende Dreiteilung der maßgeblichen Gruppen in Heer, Senat und Volk ist ein bereits in der Antike geschaffenes Konstrukt ${ }^{132}$, das aus durchsichtigen Interessen vorgenommen wurde. Der antike Schematismus diente dazu, einer als eher unübersichtlich und undurchschaubar wahrgenommenen politischen Realität Struktur zu geben ${ }^{133}$ und nicht nachvollziehbare Willensbildungsprozesse zu erklären, indem man sie Kollektiven und ihnen inhärenten Normensystemen zuschrieb, denen man gemeinsame Interessen, Vorstellungen, Werte und politische Ziele unterstellte. In der Kaiserzeit blieb zudem der in der römischen Republik entwickelte Habitus senatorischen Konsenses trotz der spätrepublikanischen Verwerfungen wirksam ${ }^{134}$. Es mag sein oder liegt vielleicht sogar nahe, dass die Akteure in bestimmten Situationen diese abstrakten Kollektive generierten, indem sie in ritualisierten Abläufen Gruppenbildungen mit entsprechenden moralisch-ethischen wie politischen Zuschreibungen vor-

129 Siehe etwa Ulrich Gotter, Die Nemesis des Allgemein-Gültigen. Max Webers Charisma-Konzept und die antiken Monarchien, in: Pavlina Rychterová u.a. (Hg.), Das Charisma. Funktionen und symbolische Repräsentationen (Berlin 2008) 173-186, hier 180 (römische Monarchie als „kommunikatives Geflecht“).

${ }^{130}$ Egon Flaig, Den Kaiser herausfordern. Die Usurpation im Römischen Reich (Frankfurt am Main, New York 1992). Siehe zu kollektiven Willensbildungen und Kommunikation zwischen Institutionen sowie zu Normenkonsens ders., Ritualisierte Politik. Zeichen, Gesten und Herrschaft im Alten Rom (Göttingen 2003) 222-231. Parallel und unabhängig von Flaig erschien Julia Sünskes Thompson, Demonstrative Legitimation von Kaiserherrschaft im Epochenvergleich. Zur politischen Macht des stadtrömischen Volkes (Stuttgart 1993). Vgl. zustimmend zu Flaig exempli gratia Jochen Martin, Der Verlust der Stadt, in: Christian Meier (Hg.), Die okzidentale Stadt nach Max Weber (München 1994) 95-114, hier 108; Gregor Weber, Kaiser, Träume und Visionen in Prinzipat und Spätantike (Stuttgart 2000) 17f.; Olivier Hekster, Commodus. An Emperor at the Crossroads (Amsterdam 2002) 17; Seelentag, Taten (wie Anm. 4) 17-29; Hans Schlange-Schöningen, Augustus (Darmstadt 2005) 145; Gotter, Nemesis (wie Anm. 129) 179 f.

${ }^{131}$ Siehe hierzu die Kritik bei Martin Zimmermann, Herodians Konstruktion der Geschichte und sein Blick auf das stadtrömische Volk, in: Ders. ( Hg.), Geschichtsschreibung und politischer Wandel im 3. Jahrhundert n. Chr. (Stuttgart 1999) 119-143, bes. 127-143. Siehe hierzu demnächst auch Katja Kröss, Die plebs urbana in der römischen Kaiserzeit (Diss. München). Vgl. bereits die differenzierte Darstellung bei Zvi Yavetz, Plebs and Princeps (New Brunswick u.a. ${ }^{2}$ 1988).

${ }^{132}$ Siehe z.B. Werner Eck u.a., Das Consultum de Cn. Pisone patre (München 1996) 247-254 (zu den Danksagungen des Senats an den ordo equester, die plebs und die Soldaten, wobei freilich in dem Text deutlich durchscheint, dass die Gruppen nicht als homogene Massen betrachtet wurden und der Danksagung für willfährige Zustimmung eine kompliziertere Realität gegenüberstand, die zumindest Tacitus noch erkennen lässt). Vgl. auch Zimmermann, Kaiser (wie Anm. 7) 171-203 zur historiographischen und fiktiven Konstruktion, nach der Pescennius Niger der Kaiser der plebs, Septimius Severus der Kaiser der Soldaten und Didius Iulianus der Kaiser des Senats waren.

${ }^{133}$ Siehe zur Einheitlichkeit des Senats als Fassade Eich, Aristokratie (wie Anm. 101) 129-132, der eine Studie zu den möglichen Kollektivinteressen der Senatoren zu Recht als Desiderat bezeichnet (131).

${ }^{134}$ Karl-Joachim Hölkeskamp, Rekonstruktionen einer Republik (München 2004) 85-92. 
nahmen. Dennoch verlief der Alltag politischer Willensbildung und die Kommunikation darüber, was einen guten Kaiser ausmachte und ob ein bestimmter Kaiser zu diesen zu zählen sei, nicht linear zwischen Herrscher und Akteuren, die sich als Repräsentanten einer der drei Gruppen verstanden. Diese Vorstellung ist schon deshalb kaum mit den tatsächlichen Verhältnissen in Einklang zu bringen, da allein die plebs urbana eine sozial derart heterogene Gruppe darstellte, deren Interessen kaum auf einen gemeinsamen Nenner zu bringen waren. Ähnliches gilt für den mit Blick auf Status, Prestige und Einfluss sehr differenzierten Senatorenstand ${ }^{135}$ oder das Heer, dessen Soldaten zudem in verschiedenen Teilen des Reiches vielfältig mit der Zivilbevölkerung verwachsen waren, und das jedenfalls nicht als abgeschlossene Institution zu begreifen ist ${ }^{136}$.

Trotz der vielfältigen Anregungen, die von dem Entwurf eines Akzeptanzsystems ausgehen, stellen die Mechanismen der Kommunikation weiterhin ein echtes Forschungsdesiderat dar bzw. sie haben diese Lücke, die bereits Alföldi gesehen hat und in seinen Forschungen nicht mehr schließen konnte, umso deutlicher zutage treten lassen. Flaig selbst hat verschiedentlich darauf hingewiesen, dass die Aushandlungsprozesse komplizierter sind, als von ihm in dem genannten Modell suggeriert wird ${ }^{137}$. In den Verhandlungen zwischen einzelnen Herrschern und den Adressaten der Repräsentation spielten zudem Vorstellungen eine Rolle, die über kollektive Identitäten, sofern diese überhaupt greifbar sind, deutlich hinausgehen. Der viel zitierte und oft von den Herrschern beschworene consensus universorum war ausdrücklich so unspezifisch angelegt, dass ganz verschiedene Teile der Bevölkerung sich in dem Begriff wiederfanden. Sie konnten sich dabei sehr unterschiedlichen Teilidentitäten zuordnen: als römische Bürger, als Senatoren, als Ritter, als Stadtbewohner, als Bauern, als Vertreter eines Kollegiums, als Mitglieder einer Nachbarschaft usw. Sieht man sich die vielfältigen Formen von Ehrungen an, die diese unterschiedlichen Gruppen und Personen an verschiedenen Orten platzierten, dann zeigt sich, wie schwierig es ist, aus dieser Summe ein kohärentes, in seinen Einzelteilen aufeinander bezogenes Ganzes zu erkennen und dies den von Flaig definierten Sektoren der Gesellschaft eindeutig zuzuweisen.

Alexander Heinemann etwa hat in seiner detaillierten Studie zur Ehrung verstorbener Kaisersöhne verdeutlicht, dass „der Komplex von Bauten und Bildern, Räumen und Ritualen“ sich als „sinnvoll aufeinander bezogenes Gefüge beschreiben (lässt), in dem einzelne Teile der Gesellschaft miteinander in Kommunikation treten“. Die Frage aber, ob es sich um „ein geschlossenes System, das aus bewussten Entscheidungen der Zeitgenossen resultiert“, handelt oder „um kontingente Begleiterscheinungen einer nur lose strukturierten Vielstimmigkeit“, sei kaum zu entscheiden ${ }^{138}$. Er plädiert, wie die bereits mehrfach zitierte Marianne Bergmann, eher für ein unsystematisches Vorgehen der ehrenden Gruppen, das aber durchaus Sinnhaftigkeit produzieren möchte, auch wenn diese nur darin besteht, durch erreichtes Wohlwollen des Herrschers reziprok persönlichen Einfluss auszubauen. Hinter diesen Überlegungen steht demnach ein Aspekt des Prinzipats, der bei der mittlerweile breiten Adaptation des Akzeptanzgedankens in Teilen der aktuellen Forschung immer

${ }^{135}$ Siehe z.B. die prosopographische Arbeit von André Chastagnol, Le sénat romain à l'époque impériale (Paris 1992).

${ }^{136}$ Hierzu mit weiteren Hinweisen Martin Zimmermann, „... als wären sie selbst dabei gewesen“. Antike Kriegslandschaften in Bild und Text, in: Georg Schildt, Anton Schindling (Hg.), Kriegserfahrungen - Krieg und Gesellschaft in der Neuzeit. Neue Horizonte der Forschung (Paderborn u.a. 2009) 41-81.

${ }_{137}$ Z.B. Egon Flaig, Keine Performanz ohne Norm - keine Norm ohne Wert. Das Problem der zwingenden Gesten in der römischen Republik, in: Andreas Haltenhoff u.a. (Hg.), Römische Werte als Gegenstand der Altertumswissenschaft (Leipzig 2005) 209-221, hier 219.

${ }^{138}$ Heinemann, Archäologie (wie Anm. 125) 106. 
mehr in den Hintergrund tritt, nämlich die ungeheure Machtfülle, die der Kaiser trotz aller kommunikativen Elemente herrscherlicher Repräsentation inne hatte. Bereits Andreas Alföldi hat in seinen Arbeiten zur monarchischen Repräsentation davor gewarnt, den autokratischen Charakter des Systems zu unterschätzen, zumal gerade die „moderne Wertung der augusteischen Monarchie den wahren Sachverhalt oft verdunkelt ${ }^{\text {"139. }}$. Der Kaiser hatte die militärischen Gewaltmittel in seiner Hand und stand unbestritten an der Spitze einer differenzierten Hierarchie. Neben ihm gab es de facto keine Gleichen.

Auf der politischen Ebene spielte dennoch die Einbindung gerade der führenden Gruppen eine besonders gewichtige Rolle. Der Kaiser musste, wie wir gesehen haben, zum einen Repräsentationsformen entwickeln, die seine herausragende Stellung adäquat wiedergaben. Zum anderen musste er darauf bedacht sein, diese Formen so geschickt einzusetzen, dass suggeriert werden konnte, er besitze gar keine herausragende Stellung, sondern diese sei nur als Weiterentwicklung bereits bestehender Institutionen zu verstehen. Dieses Paradoxon führte zwangsläufig dazu, dass der Prinzipat einen relativ geringen Grad an spezifischer Institutionalisierung erreichte und in großem Umfang an traditionellen Mustern orientiert blieb oder zumindest so tat, als sei er Bewährtem verpflichtet. Diese mangelnden Festlegungen erzeugten einen ungeheuren Spielraum bei der Ausfüllung der kaiserlichen Rolle. Dies konnte ins Positive ausschlagen (wie für Trajan behauptet) oder sich komplett zum Negativen wenden (wie bei Caligula zu studieren), wenn die Machtfülle tatsächlich ausgespielt wurde. Entscheidend war, dass die einzelnen Herrscher sich wie Schauspieler in sehr unterschiedlichen Rollenmustern zu bewegen hatten. Die beispielsweise für Trajan konstatierten Rollenwechsel ${ }^{140}$ lassen erkennen, dass diese einzelnen Rollen signalisierten, dass der Herrscher die alten Formen symbolischer Kommunikation fortführte. Die einzelnen Rollenmuster hatten freilich exemplarischen Charakter: Der Kaiser musste anzeigen, dass er stellvertretend für alle handelte und immer im Sinne aller agierte. Entscheidend war der Kommunikationszusammenhang, in dem die Beteiligten durch Partizipation und aktive Teilhabe ihre spezifischen Vorteile und ihren persönlichen politischen wie sozialen Nutzen ziehen konnten. In dieser umfassenden Suggestion von Teilhabe lag ein Geheimnis der innenpolitischen Stabilität, die erst in Folge neuartiger Bedrohungen am Ende des 2. und vor allem im 3. Jahrhundert brüchig wurde.

${ }^{139}$ Alfoldi, Repräsentation (wie Anm. 1) 25.

${ }^{140}$ Ronning, Herrscherpanegyrik (wie Anm. 71) 106-115. 
